TRANSACTIONS OF THE

AMERICAN MATHEMATICAL SOCIETY

Volume 357, Number 11, Pages 4349-4377

S 0002-9947(05)04003-1

Article electronically published on June 21, 2005

\title{
UNIFORM PROPERTIES OF RIGID SUBANALYTIC SETS
}

\author{
LEONARD LIPSHITZ AND ZACHARY ROBINSON
}

\begin{abstract}
In the context of rigid analytic spaces over a non-Archimedean valued field, a rigid subanalytic set is a Boolean combination of images of rigid analytic maps. We give an analytic quantifier elimination theorem for (complete) algebraically closed valued fields that is independent of the field; in particular, the analytic quantifier elimination is independent of the valued field's characteristic, residue field and value group, in close analogy to the algebraic case. This provides uniformity results about rigid subanalytic sets. We obtain uniform versions of smooth stratification for subanalytic sets and the Łojasiewicz inequalities, as well as a unfiorm description of the closure of a rigid semianalytic set.
\end{abstract}

\section{INTRODUCTION}

A rigid subanalytic set is a Boolean combination of images of rigid analytic maps. Techniques of quantifier elimination have proved useful in the theory of rigid subanalytic sets. In this paper, we give an analytic elimination theorem for algebraically closed valued fields that is independent of the field; in particular, the quantifier elimination is independent of the valued field's characteristic, residue field and value group. This uniform analytic elimination theorem provides uniformity results about rigid subanalytic sets. We obtain, for example, uniform versions of the smooth stratification of subanalytic sets and the Łojasiewicz inequalities.

The use of quantifier elimination in the theory of subanalytic sets is due to Denef and van den Dries $\left[\mathrm{DD}\right.$, who combined the algebraic elimination theorem for $\mathbb{Q}_{p}$ of Macintyre [Mac1] with parameterized Weierstrass Preparation to prove an analytic quantifier elimination theorem for the $p$-adic integers $\mathbb{Z}_{p}$. They used this as the basis for the theory of $p$-adic subanalytic sets. Combining the algebraic elimination theorem for algebraically closed valued fields of A. Robinson [Ro] and Weispfenning [W] with parameterized Weierstrass Preparation for rings of separated power series, [L, [LR1] - LR7] gave a rigid analytic quantifier elimination and a theory of rigid subanalytic sets. See also the papers [S1]-S5] of Schoutens. For a survey of these and other results, see D2.

The rigid analytic elimination has the following uniform property. Let $K \subset F$ be an extension of complete, non-Archimedean valued fields, where $F$ is algebraically closed. Suppose $X_{1}, \ldots, X_{n}$ are $F$-analytic varieties given as zero-sets of $K$-analytic power series (see [LR5]). Any Boolean combination of images of the $X_{i}$ under coordinate projection is defined by a quantifier-free $L_{a n}^{D}$ formula that involves only

Received by the editors March 7, 2003.

2000 Mathematics Subject Classification. Primary 03C10, 32P05, 32B20; Secondary 26E30, $03 \mathrm{C} 98$.

This work was supported in part by NSF grant number DMS 0070724.

(C)2005 American Mathematical Society 4349

Reverts to public domain 28 years from publication 
$K$-analytic power series. Furthermore, this formula does not depend on the particular choice of algebraic extension, $F$. Indeed, many properties such as a smooth stratification and Łojasiewicz inequalities of such a Boolean combination, i.e., a subanalytic set, are independent of the field $F$. In other words, the theory of $K$-subanalytic sets is uniform in $F$.

However, none of the above-mentioned theories of subanalytic sets fully exploited the uniformity of the algebraic elimination. Consider a system $(*)$ that is a Boolean combination of conditions of the form

$$
|p(x, y)| \bowtie|q(x, y)|,
$$

where $\bowtie \in\{=,<\}, p, q \in \mathbb{Z}[x, y]$, and $x$ and $y$ are several variables. It follows from the algebraic elimination theorem that there are polynomials $p^{\prime}, q^{\prime} \in \mathbb{Z}[x]$ and a Boolean combination $(* *)$ of conditions of the form

$$
\left|p^{\prime}(x)\right| \bowtie\left|q^{\prime}(x)\right|,
$$

$\bowtie \in\{=,<\}$, such that for every algebraically closed valued field $F,(* *)$ defines the image under projection from $F^{n} \times F^{m}$ to $F^{n}$ of the set defined by $(*)$. Conditions (**) depend neither on the field $F$ nor its characteristic. In this paper, we give a full analytic analog, Theorem 4.2, of this uniform algebraic elimination theorem.

Parameterized Weierstrass Preparation provided a key step in going from algebraic to analytic elimination theory. Where Denef and van den Dries used the prime $p$ of the valuation ring of $\mathbb{Q}_{p}$ (which equals the characteristic of the residue field), we used variables $\rho$, interpreted to range over the maximal ideal of the valuation ring, to witness the inequalities arising in Weierstrass Preparation. This syntactic uniformity in our language suggested that it would yield an approach to a uniform analytic elimination theorem for all complete, algebraically closed, non-Archimedean valued fields in a suitable language.

While there are elements of $\mathbb{Z}[[\xi]] \backslash \mathbb{Z}[\xi]$ that belong to the ring $\mathbb{Z}_{p}\langle\xi\rangle$ of strictly convergent power series for all $p$, for example, $f=\sum_{n=1}^{\infty} n ! \xi^{n}$, no such power series is strictly convergent in equicharacteristic zero, nor does it have a bound, uniform in $p$, on the number of zeros in the set $|\xi|_{p} \leq 1$. (The lack of a uniform bound can be seen by observing for each $p$ that $f$ is Weierstrass regular of degree $p-1$ in the ring $\mathbb{Z}_{p}\langle\xi\rangle$.) If, however, we view $f$ as a separated power series and restrict its domain to $|\xi|<1$ (i.e., change $\xi$ to $\rho$ ), we see that $f(\rho)$ is Weierstrass regular of degree 1 in the ring $\mathbb{Z}[[\rho]]$, independent of the prime $p$. In general, for any element $f \in \mathbb{Z}[\xi][[\rho]]$, there is a uniform bound on the degree of (pre)regularity, even in parameters (Corollary 3.8).

Since the functions in $\mathbb{Z}[\xi][[\rho]]$ are separated power series, the geometry of sets defined using them is known to be well behaved for each fixed ultrametric field (see [LR5]). Indeed, we shall show that sets defined using such functions are uniformly well behaved. Such sets, for example, have smooth stratifications defined over $\mathbb{Z}[\xi][[\rho]]$ uniformly for all complete, algebraically closed, non-Archimedean valued fields $F$ (see Corollary 4.6).

In $\mathrm{vdD}$, van den Dries introduced the concept of a valued field with analytic $\mathbb{Z}[[t]]$-structure, where $t$ is interpreted as a uniformizing parameter of the valuation ring. This provides a vehicle for extending the uniform algebraic results of Pas [Pas] and Macintyre Mac2 to the category of (discretely valued) Henselian valuation rings with analytic structure. He suggests that a similar development could be 
carried out in the non-discretely valued (algebraically closed) case using rings of separated power series. This is our starting point in Section 2.

In Section 2, we establish notational conventions, introduce some power series rings, set up the first-order languages that are used throughout the rest of the paper, and introduce the axioms for the uniform theory of valued fields with analytic structure. In Section 3, we show how Weierstrass Division works in a uniform context, and prove the corresponding division theorem as well as other technical results that are needed for the uniform analytic elimination theorem. In Section 4, we prove the uniform elimination theorem (Theorem 4.2) and give some applications, such as existence of prime models (Corollary 4.5) and uniform smooth stratification (Corollary 4.6). In Section 5, we give the axioms for the theory of valued fields with analytic structure that contain a fixed complete field $K$, and discuss certain nonstandard models of these theories. In Section 6, we use the non-standard models of Section 5 to give an improved treatment of the rigid analytic Łojasiewicz inequalities, and to show that these inequalities are uniform in various ways. In Section 7, we use the Łojasiewicz inequalities to give a uniform and elementary proof that the closure of a rigid semianalytic set is also semianalytic. This extends the results of Huber $[\mathrm{H}$, Liu $[\mathrm{Liu}$ and Schoutens $[\mathrm{S5}]$ and avoids the use of valuation spectra and resolution of singularities. In Section 8, we give an alternative proof of the curve selection theorem of Huber $[\underline{\mathrm{H}}]$.

\section{Definitions and notation}

The basic open domains of non-Archimedean analysis over an algebraically closed (complete) valued field $F$ are the unit polydiscs $\left(F^{\circ}\right)^{m} \times\left(F^{\circ}\right)^{n}$, where $F^{\circ}$ is the valuation ring of the valued field $F$ and $F^{\circ \circ}$ is the maximal ideal of $F^{\circ}$; i.e.,

$$
\begin{aligned}
F^{\circ} & :=\{x \in F:|x| \leq 1\}, \\
F^{\circ \circ} & :=\{x \in F:|x|<1\} .
\end{aligned}
$$

(In Sections 4 and 5, we extend this to algebraically closed, valued fields $F$ that are elementarily equivalent to a complete field.) Taking the quotient, we obtain the residue field $\widetilde{F}:=F^{\circ} / F^{\circ 0}$. In this paper we employ the convention that $\xi=\left(\xi_{1}, \ldots, \xi_{m}\right)$ are variables that range over $F^{\circ}$ and $\rho=\left(\rho_{1}, \ldots, \rho_{n}\right)$ are variables that range over $F^{\circ \circ}$. We also use the convention for a normed ring $R$ that

$$
\begin{aligned}
R^{\circ} & :=\{x \in R:|x| \leq 1\}, \\
R^{\circ \circ}: & =\{x \in R:|x|<1\} .
\end{aligned}
$$

The ring

$$
T_{m}(K):=\left\{\sum_{\mu \in \mathbb{N}^{m}} a_{\mu} \xi^{\mu}: \lim _{|\mu| \rightarrow \infty} a_{\mu}=0\right\}
$$

of strictly convergent power series consists of those power series that converge on the closed unit polydiscs $\left(F^{\circ}\right)^{m}$, where $F$ is as above and $K$ is a complete subfield. As in [LR5, while the models $F^{\circ}$ are algebraically closed, we do not require the coefficient field $K$ to be algebraically closed: for a fixed coefficient field $K$, the elimination theorem of [LR5] is uniform in $F \supset K$. In the model theory of algebraically closed valued fields with analytic structure, it is convenient to have well-behaved rings of analytic functions on the polydiscs $\left(F^{\circ}\right)^{m} \times\left(F^{\circ \circ}\right)^{n}$. These are provided by the 
rings $S_{m, n}(E, K)$ of separated power series,

$$
S_{m, n}(E, K):=K \otimes_{K^{\circ}} \bigcup_{B \in \mathcal{B}} B\langle\xi\rangle[[\rho]],
$$

where $\mathcal{B}$ is the collection of complete, quasi-Noetherian rings consisting of the rings

$$
B:=E\left[a_{i}: i \in \mathbb{N}\right]_{\left\{a \in E\left[a_{i}: i \in N\right]:|a|=1\right\}}^{\wedge}
$$

and where $E$ is a fixed complete, quasi-Noetherian subring of $K^{\circ}$ (for example, $E$ may be a field or DVR contained in $\left.K^{\circ}\right),\left\{a_{i}\right\}_{i \in \mathbb{N}}$ is a zero-sequence of $K^{\circ}$, and $\wedge$ denotes completion in the valuation topology (see [LR4]).

Note that $K[[\xi, \rho]] \supset S_{m, n} \supset T_{m}$. Let $f=\sum a_{\mu \nu} \xi^{\mu} \rho^{\nu} \in S_{m, n}$. The function

$$
f \mapsto \max _{\mu \nu}\left|a_{\mu \nu}\right| \in|K|
$$

is called the Gauss norm, which is a multiplicative norm on $S_{m, n}$ extending the norm on $K$.

Let $\overline{\mathbb{Q}}$ denote the algebraic closure of $\mathbb{Q}$ and let $\overline{\mathbb{Z}}$ denote the integral closure of $\mathbb{Z}$ in $\overline{\mathbb{Q}}$. The rings

$$
\begin{aligned}
U_{m, n}(\mathbb{Z}): & =\mathbb{Z}[\xi][[\rho]], \\
U_{m, n}(\mathbb{Z}, \overline{\mathbb{Z}}): & =\bigcup_{\substack{\mathbb{Z} \subset E \subset \overline{\mathbb{Z}} \\
\text { finitely generated }}} E[\xi][[\rho]], \\
U_{m, n}(\mathbb{Z}, \mathbb{Q}): & =\bigcup_{\ell \in \mathbb{N}} \mathbb{Z}\left[\frac{1}{\ell}\right][\xi][[\rho]] \text { and } \\
U_{m, n}(\mathbb{Z}, \overline{\mathbb{Q}}):= & \bigcup_{\substack{\mathbb{Z} \subset E \subset \overline{\mathbb{Z}} \\
\text { finitely generated } \\
\ell \in \mathbb{N}}} E\left[\frac{1}{\ell}\right][\xi][[\rho]]
\end{aligned}
$$

have properties analogous to $S_{m, n}$ and can be interpreted uniformly as analytic functions on $\left(F^{\circ}\right)^{m} \times\left(F^{\circ \circ}\right)^{n}$ as follows. Fix the fields $K \subset F$. The natural homomorphism $\mathbb{Z} \rightarrow K \subset F$ extends, coefficient-wise, to a homomorphism

$$
\sigma: U_{m, n}(\mathbb{Z}) \rightarrow S_{m, n}^{\circ}(E, K)
$$

where $E$ is the completion of $\sigma(\mathbb{Z})$ in $K$. This is a monomorphism if Char $K=0$; otherwise the kernel is generated by $p=$ Char $K$. If $K$ contains the algebraic closure of its prime field, then $\sigma$ extends to a homomorphism of $U_{m, n}(\mathbb{Z}, \overline{\mathbb{Z}})$ into $S_{m, n}^{\circ}(E, K)$. If $K$ is an equicharacteristic zero field, then $\sigma$ extends to a monomorphism of $U_{m, n}(\mathbb{Z}, \mathbb{Q})$ into $S_{m, n}^{\circ}(E, K)$; if in addition $K$ contains the algebraic closure of its prime field, then $\sigma$ extends to a monomorphism of $U_{m, n}(\mathbb{Z}, \overline{\mathbb{Q}})$ into $S_{m, n}^{\circ}(E, K)$. Hence for any complete field $F$ the power series in $U_{m, n}(\mathbb{Z})$ represent, via $\sigma$, analytic functions on $\left(F^{\circ}\right)^{m} \times\left(F^{\circ \circ}\right)^{n}$. The homomorphisms $\sigma$ (and their extensions) allow us to uniformly represent analytic functions on $\left(F^{\circ}\right)^{m} \times\left(F^{\circ \circ}\right)^{n}$.

As usual $L=\langle 0,1,+, \cdot,|\cdot|, \overline{0}, \overline{1},-,<\rangle$ denotes the three-sorted language of multiplicatively valued rings. The three sorts are: the valuation ring, its maximal ideal and the value semigroup. We suppress the predicates for the sorts, and we will usually write 0,1 and · for the elements $\overline{0}, \overline{1}$ of the value semigroup and for multiplication in this semigroup; no confusion should arise. The standard interpretations for $L$ that we have in mind are the valuation rings $F_{a l g}^{\circ}$, where $F$ is a complete valued field and $F_{\text {alg }}$ is its algebraic closure. We may enrich the language 
by adding function symbols for all the elements of $U:=\bigcup_{m, n} U_{m, n}(\mathbb{Z})$. We denote this extended language as $L_{a n}(U)$. Function symbols and constant symbols are interpreted in the obvious way modulo $p$ under the homomorphism $\sigma$. When, as in [LR5], the fields $F$ are extensions of a fixed complete, valued field $K$, it is convenient to use a language $L_{a n}(K)$, obtained from $L$ by adding symbols for the elements of $S_{m, n}^{\circ}(E, K)$, or certain of its subrings, as in [LR5].

More generally, the $L_{a n}(U)$-structures in which we shall be interested are the valuation rings $F^{\circ}$ on which the functions of $U$ are defined and satisfy all the power series identities of $U$ (see Definition 2.1(ii)). For any field $F$, complete in a nontrivial non-Archimedean absolute value $|\cdot|: F \rightarrow \mathbb{R}_{+}$, the elements of $U_{m, n}(\mathbb{Z})$ naturally define functions on $\left(F^{\circ}\right)^{m} \times\left(F^{\circ \circ}\right)^{n}$ and satisfy the power series identities due to the convergence of their power series expansions. There are, however, also natural $L_{a n}(K)$-structures $F^{\circ}$ which are not complete, but on which the functions of $S$ can be defined so as to satisfy all the power series identities of $S$, as in Definition 5.1(ii).

The language $L_{a n}^{D}(U)$ is obtained from $L_{a n}(U)$ by adding two more function symbols, $D_{0}: F^{\circ} \times F^{\circ} \rightarrow F^{\circ}$ and $D_{1}: F^{\circ} \times F^{\circ} \rightarrow F^{\circ \circ}$, for restricted division:

$$
\begin{aligned}
& D_{0}(x, y):= \begin{cases}x / y & \text { if }|x| \leq|y| \neq 0, \\
0 & \text { otherwise, }\end{cases} \\
& D_{1}(x, y):= \begin{cases}x / y & \text { if }|x|<|y|, \\
0 & \text { otherwise. }\end{cases}
\end{aligned}
$$

Observe that $D_{0}\left(t_{1}, t_{2}\right)$ is a term of the valuation ring sort, and that $D_{1}\left(t_{1}, t_{2}\right)$ is a term of the maximal ideal sort. We will often refer to an $L_{a n}^{D}$-term of either of these sorts (i.e., not the value semi-group sort) as a D-function. In other words, a $D$-function is a term obtained by composing $D_{0}, D_{1}$ and the power series. Strictly speaking, a $D$-function $f$ is a term which is interpreted via $\sigma$ as a function in each model $F^{\circ}$ (of the theory described either by Definition 2.1 or by Definition 5.1).

Let $F$ be a complete valued field that contains the algebraic closure of its prime field and fix $f \in U_{m, n}(\mathbb{Z}, \overline{\mathbb{Z}})$. Since the coefficients of $f$ lie in a fixed finite extension of $\mathbb{Z}$, there is a term $t \in L_{a n}(U)$ and $c_{1}, \ldots, c_{n} \in \overline{\mathbb{Z}}$ such that

$$
t\left(c_{1}, \ldots, c_{k}, \xi_{1}, \ldots, \xi_{m}, \rho_{1}, \ldots, \rho_{n}\right)
$$

represents $\sigma(f)$ on $\left(F^{\circ}\right)^{m} \times\left(F^{\circ \circ}\right)^{n}$.

Let $F$ be a complete, equicharacteristic zero, valued field that contains the algebraic closure of its prime field and let $f \in U_{m, n}(\mathbb{Z}, \overline{\mathbb{Q}})$. Then there is an $L_{a n}^{D}(U)$-term $t\left(D_{0}\left(c_{1}, \ell\right), \ldots, D_{0}\left(c_{k}, \ell\right), \xi_{1}, \ldots, \xi_{m}, \rho_{1}, \ldots, \rho_{n}\right)$, with $c_{1}, \ldots, c_{k} \in \overline{\mathbb{Z}}$ and $\ell \in \mathbb{Z}$, that represents $\sigma(f)$. Indeed, $t$ represents $\sigma(f)$ as long as Char $K \nmid \ell$.

We now introduce the axioms $\mathcal{T}_{a n}(U)$ in the (characteristic independent) language $L_{a n}(U)$ for the uniform theory of algebraically closed valued fields with analytic structure. In Section 5, we consider the analogous theory $\mathcal{T}_{\text {an }}(S)$ for fields that contain a fixed complete field $K$.

(2.1) Definition. The axioms in the language $L_{a n}(U)$ for the uniform theory of algebraically closed fields with analytic structure, $\mathcal{T}_{a n}(U)$, are:

(i) the axioms, in the 3-sorted language $L$, for the valuation ring of an algebraically closed, non-Archimedean, non-trivially valued field, and 
(ii) all identities of the form

$$
f=F\left(g_{1}, \ldots, g_{m}, h_{1}, \ldots, h_{n}\right),
$$

where $F \in U_{m, n}, g_{1}, \ldots, g_{m} \in U_{M, N}$ and $h_{1}, \ldots, h_{n} \in(\rho) \cdot U_{M, N}$. Note that this includes all Weierstrass data (see Section 3) and the diagram of the ring $U=\bigcup_{m, n} U_{m, n}$.

The axioms $\mathcal{T}_{a n}^{D}(U)$ in the language $L_{a n}^{D}$ are those of $\mathcal{T}_{a n}(U)$ together with the obvious axioms defining $D_{0}$ and $D_{1}$ as in Section 2 .

\section{WEIERSTRASS PREPARATION AND DIVISION THEOREMS}

In this section, we show that the rings $U_{m, n}$ satisfy Weierstrass Division Theorems similar to those satisfied by the rings $S_{m, n}$. Indeed, these theorems are all a consequence of the division theorems for the rings $A[[\rho]]$ and $A\langle\xi\rangle$, where $A$ is a complete, linearly topologized ring (see Theorem 3.2). We define 'preregularity' (Definition 3.5) for the rings $U_{m, n}$ and we show that the degree of preregularity and the Weierstrass data in $U_{m, n}$ are preserved under the map $\sigma$, discussed in Section 2 , by means of which we interpret the elements of $U_{m, n}$ as analytic functions in various valued fields (see Proposition 3.4 and Corollary 3.8).

For example, the element $f=4 \rho+\rho^{2} \in U_{0,1}(\mathbb{Z})$ is not regular of any degree in the ring $U_{0,1}(\mathbb{Z})$. However the image of $f$ in $S_{0,1}\left(\mathbb{Z}_{p}, \mathbb{Q}_{p}\right)$ is regular of degree 1 when $p \neq 2$, and the image of $f$ in $S_{0,1}\left(\mathbb{Z}_{2}, \mathbb{Q}_{2}\right)$ is regular of degree 2. More precisely, although $f$ is not preregular of any degree, in every ultrametric field the following is true:

$$
\left(|4|<1 \wedge f=D_{1}(4,1) \rho+\rho^{2}\right) \vee\left(|4| \geq 1 \wedge f=4\left(\rho+D_{0}(1,4) \rho^{2}\right) .\right.
$$

Hence if we let $F_{1}:=\lambda \rho+\rho^{2}$ and $F_{2}:=\rho+\xi \rho^{2}$, then $F_{1}$ is preregular of degree $(0,2)$ and $F_{2}$ is preregular of degree $(0,1)$, and in every ultrametric field $K$

$$
\sigma(f)=F_{1}\left(D_{1}(4,1), \rho\right) \text { or } \sigma(f)=4 F_{2}\left(D_{0}(1,4), \rho\right) .
$$

We introduce some conventions for Theorem 3.2, Weierstrass Division. Let $A$ be a ring, equipped with a linear topology (for example, the $I$-adic topology for a fixed ideal $I$ of $A$ ). We will be interested primarily in the cases that $A=U_{m, n-1}(\mathbb{Z})$ or $A=U_{m-1, n}(\mathbb{Z})$ and $I=\left(\rho^{\prime}\right)$ or $I=(\rho)$, where $\rho^{\prime}=\left(\rho_{1}, \ldots, \rho_{n-1}\right)$, and that $A=S_{m, n-1}^{\circ}$ or $S_{m-1, n}^{\circ}$ and $I=\left(\varepsilon, \rho^{\prime}\right)$ or $(\varepsilon, \rho)$, where $\varepsilon \in \mathbb{Z}$ is prime.

Consider the ring $A\left[\left[\rho_{n}\right]\right]$ of formal power series in the variable $\rho_{n}$, and the ring

$$
A\left\langle\xi_{m}\right\rangle:=\left\{\sum_{i=0}^{\infty} a_{i} \xi_{m}^{i}: a_{i} \in A, \lim _{i \rightarrow \infty} a_{i}=0\right\}
$$

of strictly convergent power series in the variable $\xi_{m}$. Let $A$ be linearly topologized by a family of ideals $I_{\nu}$ of $A$. We endow $A\left\langle\xi_{m}\right\rangle$ and $A\left[\left[\rho_{n}\right]\right]$ with the linear topologies generated respectively by $I_{\nu} A\left\langle\xi_{m}\right\rangle$ and by $\left(I_{\nu}, \rho_{n}^{i}\right) A\left[\left[\rho_{n}\right]\right]$. Call an element $f$ topologically nilpotent iff $\lim _{i \rightarrow \infty} f^{i}=0$.

(3.1) Definition.

(i) An element $f \in A\left[\left[\rho_{n}\right]\right]$ is regular of degree $d$ iff

$$
f=\sum_{i=0}^{d-1} t_{i} \rho_{n}^{i}+\rho_{n}^{d}\left(1+s \rho_{n}\right)
$$

for some $s \in A\left[\left[\rho_{n}\right]\right]$ and $t_{i} \in A$ topologically nilpotent. 
(ii) An element $f \in A\left\langle\xi_{m}\right\rangle$ is regular of degree $d$ iff

$$
f=t+\xi_{m}^{d}+a_{1} \xi_{m}^{d-1}+\ldots+a_{d}
$$

with $t \in A\left\langle\xi_{m}\right\rangle$ topologically nilpotent and $a_{1}, \ldots, a_{d} \in A$.

As in [Ba1, Section 2.2] and [Ba2, Section 1.2], or [LR4, Section 2.3], we have the following.

(3.2) Weierstrass Preparation and Division Theorems. Let $A$ be a ring, complete in a linear topology.

(i) Let $f, g \in A\left\langle\xi_{m}\right\rangle$ with $f$ regular of degree $d$. Then there are uniquely determined elements $q \in A\left\langle\xi_{m}\right\rangle$ and $r_{0}, \ldots, r_{d-1} \in A$ such that

$$
g=q f+\sum_{i=0}^{d-1} r_{i} \xi_{m}^{i} .
$$

In particular, taking $g=\xi_{m}^{d}$, we have

$$
f=u \cdot\left(\xi_{m}^{d}+a_{1} \xi_{m}^{d-1}+\ldots+a_{d}\right)
$$

for uniquely determined $a_{1}, \ldots, a_{d} \in A$ and unit $u \in A\left\langle\xi_{m}\right\rangle$, where $u=1+t$ with $t \in A\left\langle\xi_{m}\right\rangle$ topologically nilpotent.

(ii) Let $f, g \in A\left[\left[\rho_{n}\right]\right]$ with $f$ regular of degree $d$. Then there are uniquely determined elements $q \in A\left[\left[\rho_{n}\right]\right]$ and $r_{0}, \ldots, r_{d-1} \in A$ such that

$$
g=q f+\sum_{i=0}^{d-1} r_{i} \rho_{n}^{i}
$$

In particular, taking $g=\rho_{n}^{d}$, we have

$$
f=u \cdot\left(\rho_{n}^{d}+a_{1} \rho_{n}^{d-1}+\ldots+a_{d}\right),
$$

for uniquely determined topologically nilpotent $a_{1}, \ldots, a_{d} \in A$ and unit $u \in$ $A\left[\left[\rho_{n}\right]\right]$, where $u=1+t$ with $t \in A\left[\left[\rho_{n}\right]\right]$ topologically nilpotent.

The rings $U_{m, n}$ and $S_{m, n}$ satisfy the following Weierstrass Preparation and Division Theorems. Recall that we have endowed $U_{m, n}(\mathbb{Z})$ (respectively $S_{m, n}^{\circ}(E, K)$ ) with the $I$-adic topology, where $I=(\rho)$ (respectively, $I=(\varepsilon, \rho)$ with $|\varepsilon|<1$ ).

(3.3) Corollary. Let $f, g \in U_{m, n}(\mathbb{Z})$ (respectively, $S_{m, n}^{\circ}(E, K)$ ), and let $\xi^{\prime}=$ $\left(\xi_{1}, \ldots, \xi_{m-1}\right)$ and $\rho^{\prime}=\left(\rho_{1}, \ldots, \rho_{n-1}\right)$.

(i) Suppose $f$ is regular in $\xi_{m}$ of degree $d$. Then there are uniquely determined elements $q \in U_{m, n}(\mathbb{Z})$ (respectively, $S_{m, n}^{\circ}(E, K)$ ) and $r_{0}, \ldots, r_{d-1} \in$ $U_{m-1, n}(\mathbb{Z})$ (respectively, $S_{m-1, n}^{\circ}(E, K)$ ) such that

$$
g=q f+\sum_{i=0}^{d-1} r_{i} \xi_{m}^{i}
$$

In particular, taking $g=\xi_{m}^{d}$, we have

$$
f=u \cdot\left(\xi_{m}^{d}+a_{1} \xi_{m}^{d-1}+\ldots+a_{d}\right)
$$

for uniquely determined elements $a_{1}, \ldots, a_{d}$ of $U_{m-1, n}(\mathbb{Z})$ (respectively, of $S_{m-1, n}^{\circ}(E, K)$ ) and unit $u \in U_{m, n}(\mathbb{Z})$ (respectively, $\left.S_{m, n}^{\circ}(E, K)\right)$, where $u=1+t$ with $t \in U_{m, n}(\mathbb{Z})$ (respectively, $S_{m, n}^{\circ}(E, K)$ ) topologically nilpotent. 
(ii) Suppose $f$ is regular of degree $d$ in $\rho_{n}$. Then there are uniquely determined elements $q \in U_{m, n}(\mathbb{Z})$ (respectively, $S_{m, n}^{\circ}(E, K)$ ) and $r_{0}, \ldots, r_{d-1} \in$ $U_{m, n-1}(\mathbb{Z})$ (respectively, $S_{m, n-1}^{\circ}(E, K)$ ) such that

$$
g=q f+\sum_{i=0}^{d-1} r_{i} \rho_{n}^{i} .
$$

In particular, taking $g=\rho_{n}^{d}$, we have

$$
f=u \cdot\left(\rho_{n}^{d}+a_{1} \rho_{n}^{d-1}+\ldots+a_{d}\right),
$$

for uniquely determined topologically nilpotent $a_{1}, \ldots, a_{d}$ of $U_{m, n-1}(\mathbb{Z})$ (respectively $S_{m, n-1}^{\circ}(E, K)$ ) and unit $u \in U_{m, n}(\mathbb{Z})$ (respectively $S_{m, n}^{\circ}(E, K)$ ), where $u=1+t$ with $t \in U_{m, n}(\mathbb{Z})$ (respectively, $S_{m, n}^{\circ}(E, K)$ ) topologically nilpotent.

Proof. (i) Endow $U_{m-1, n}(\mathbb{Z})=\mathbb{Z}\left[\xi^{\prime}\right][[\rho]]$ with the $(\rho)$-adic topology and observe that

$$
U_{m, n}(\mathbb{Z})=\mathbb{Z}[\xi][[\rho]]=U_{m-1, n}(\mathbb{Z})\left\langle\xi_{m}\right\rangle .
$$

Now take $A=U_{m-1, n}(\mathbb{Z})$ in Theorem 3.2(i).

For the assertions regarding $S_{m, n}$, find $B \in \mathcal{B}$ (where $\mathcal{B}$ is as in Section 2) such that $f, g \in B\langle\xi\rangle[[\rho]]$, endow $B\left\langle\xi^{\prime}\right\rangle[[\rho]]$ with the linear topology induced by the linear topology on $S_{m-1, n}^{\circ}$, and proceed as for $U_{m, n}$.

(ii) Endow $U_{m, n-1}(\mathbb{Z})=\mathbb{Z}[\xi]\left[\left[\rho^{\prime}\right]\right]$ with the $\left(\rho^{\prime}\right)$-adic topology. Then we may take $A=U_{m, n-1}(\mathbb{Z})=\mathbb{Z}[\xi]\left[\left[\rho^{\prime}\right]\right]$ in Theorem 3.2(ii). The assertions regarding $S_{m, n}$ are handled similarly to part (i).

Similar Weierstrass Preparation and Division Theorems also hold for the rings $U_{m, n}(\mathbb{Z}, \overline{\mathbb{Z}}), U_{m, n}(\mathbb{Z}, \mathbb{Q})$ and $U_{m, n}(\mathbb{Z}, \overline{\mathbb{Q}})$. This follows formally from Theorem 3.2 and the observation, as in Section 2, that all elements of these rings are obtained from elements of the rings $U_{m+k, n}$ for suitable $k$, by substituting some elements of $\overline{\mathbb{Z}}$ (respectively, $\mathbb{Q}$ or $\overline{\mathbb{Q}}$ ) for $\xi_{m+1}, \ldots, \xi_{m+k}$. Note, moreover, that $f \in U_{m, n}(\mathbb{Z})$ is a unit if, and only if, $f= \pm 1+g$, where $g \in(\rho) U_{m, n}(\mathbb{Z})$. Similarly, $f \in U_{m, n}(\mathbb{Z}, \overline{\mathbb{Z}})$ (respectively, $U_{m, n}(\mathbb{Z}, \mathbb{Q})$ or $\left.U_{m, n}(\mathbb{Z}, \overline{\mathbb{Q}})\right)$ is a unit if, and only if, $f=c+g$, where $c$ is a unit of $\overline{\mathbb{Z}}$ (respectively, of $\mathbb{Q}$ or $\overline{\mathbb{Q}}$ ) and $g \in(\rho) U_{m, n}(\mathbb{Z}, \overline{\mathbb{Z}}$ ) (respectively, $(\rho) U_{m, n}(\mathbb{Z}, \mathbb{Q})$ or $\left.(\rho) U_{m, n}(\mathbb{Z}, \overline{\mathbb{Q}})\right)$. Given $f \in U_{m, n}(\mathbb{Z}, \overline{\mathbb{Z}})$, there are $c_{1}, \ldots, c_{k} \in \overline{\mathbb{Z}}$ and an $F \in U_{m+k, n}(\mathbb{Z})$ such that

$$
f(\xi, \rho)=F\left(c_{1}, \ldots, c_{k}, \xi, \rho\right) .
$$

If in addition $f$ is a unit, we can write $f$ in the form $c_{0} G\left(c_{1}, \ldots, c_{k}, \xi, \rho\right)$, where $G \in U_{m+k, n}$ is a unit of $U_{m+k, n}$. Similar observations hold for $f \in U_{m, n}(\mathbb{Z}, \mathbb{Q})$ or $U_{m, n}(\mathbb{Z}, \overline{\mathbb{Q}})$ using $D$-functions as at the end of Section 2.

We wish to consider how the Weierstrass Theorems for $U_{m, n}(\mathbb{Z})$ relate to those for $\sigma\left(U_{m, n}(\mathbb{Z})\right) \subset S_{m, n}(E, K)$.

(3.4) Proposition. Let $\sigma: U_{m, n}(\mathbb{Z}) \rightarrow S_{m, n}(E, K)$ be the homomorphism induced by the homomorphism $\mathbb{Z} \rightarrow K$ of the coefficient rings. If $f \in U_{m, n}(\mathbb{Z})$ is regular in $\rho_{n}$ (respectively $\xi_{m}$ ) of degree $d$, so is $\sigma(f)$, and if $g=q f+r$ is a Weierstrass Division in $U_{m, n}(\mathbb{Z})$, then $\sigma(g)=\sigma(q) \sigma(f)+\sigma(r)$ is the corresponding Weierstrass Division of $\sigma(g)$ by $\sigma(f)$ in $S_{m, n}(E, K)$. 
Proof. The topology on $S_{m, n}(E, K)$ is induced by the $I$-adic topology on the subring $S_{m, n}^{\circ}(E, K)$, where $I$ is the ideal generated by $(\rho)$ and an element $\varepsilon \in K^{\circ \circ} \backslash\{0\}$. With the $(\rho)$-adic topology on $U_{m, n}$, the homomorphism $\sigma$ is then continuous; in particular, if $t \in U_{m, n}(\mathbb{Z})$ is topologically nilpotent, so is $\sigma(t)$. The statement about the regularity of $\sigma(f)$ follows. To complete the proof, note that Weierstrass Division data are uniquely determined.

As with the rings $S_{m, n}(E, K)$, it is not always possible to make an $f \in U_{m, n}$ regular by a Weierstrass change of variables, even after multiplying by a suitable constant (see [LR4], Example 2.3.5). The following notion (cf. [L], [LR4]) is useful in the proof of Lemma 4.1, the induction step of the analytic elimination theorem.

(3.5) Definition. Let $f(\xi, \eta, \rho, \lambda)=\sum_{\mu \nu} a_{\mu \nu}(\xi, \rho) \eta^{\mu} \lambda^{\nu} \in U_{m+M, n+N}(\mathbb{Z})$. We say that $f$ is preregular of degree $\left(\mu_{0}, \nu_{0}\right)$ iff

(i) $a_{\mu_{0} \nu_{0}}=1$,

(ii) $a_{\mu \nu}$ is topologically nilpotent for $\nu<\nu_{0}$ and all $\mu$, and

(iii) $a_{\mu \nu_{0}}$ is topologically nilpotent for $\mu>\mu_{0}$.

Let $f\left(\xi^{\prime}, \rho^{\prime}, \eta, \lambda\right)$ be preregular of degree $\left(\mu_{0}, \nu_{0}\right)$ and let $x_{i}(\xi, \rho)$ and $t_{j}(\xi, \rho)$ be $L_{a n}^{D}$-terms of sorts $F^{\circ}$ and $F^{\circ \circ}$, respectively. Then we also call

$$
f(x(\xi, \rho), t(\xi, \rho), \eta, \lambda)
$$

preregular of degree $\left(\mu_{0}, \nu_{0}\right)$. Note that every $L_{a n}^{D}$-term in which $D_{0}, D_{1}$ are not applied to any sub-term involving $\eta$ or $\lambda$ is of the form $f(x(\xi, \rho), t(\xi, \rho), \eta, \lambda)$.

Let $K$ be a complete ultrametric field, let $F \supset K$ be a complete, algebraically closed extension field (or an extension field satisfying the axioms $\mathcal{T}_{a n}(U)$ of Definition 2.1) and let

$$
\sigma: U_{m+M, n+N}(\mathbb{Z}) \rightarrow S_{m+M, n+N}(E, K)
$$

be the natural map as above. We shall say that $\sigma(f)$ is preregular of degree $\left(\mu_{0}, \nu_{0}\right)$ at $(a, b) \in\left(F^{\circ}\right)^{m} \times\left(F^{\circ \circ}\right)^{n}$ iff

(i) $a_{\mu_{0} \nu_{0}}(a, b) \neq 0$,

(ii) $\left|a_{\mu \nu}(a, b)\right| \leq\left|a_{\mu_{0} \nu_{0}}(a, b)\right|$ for all $\mu, \nu$,

(iii) $\left|a_{\mu \nu}(a, b)\right|<\left|a_{\mu_{0} \nu_{0}}(a, b)\right|$ for $\nu<\nu_{0}$ and all $\mu$, and

(iv) $\left|a_{\mu \nu_{0}}(a, b)\right|<\left|a_{\mu_{0} \nu_{0}}(a, b)\right|$ for $\mu>\mu_{0}$.

We extend this definition as above to the case that the $a_{\mu \nu}$ are $L_{a n}^{D}$-terms not involving $\eta$ or $\lambda$; i.e., the case that $f$ is an $L_{a n}^{D}$-term in which $D_{0}, D_{1}$ are not applied to any sub-term involving $\eta$ or $\lambda$.

Abusing terminology, we say that $f$ is regular of degree $\mu_{0}$ in $\eta$ (respectively, of degree $\nu_{0}$ in $\lambda$ ) iff it is preregular of degree $\left(\mu_{0}, 0\right)$ (respectively, $\left(0, \nu_{0}\right)$ ).

(3.6) Remark. (i) Observe that if $f \in U_{m+M, n+N}(\mathbb{Z})$ is regular of degree $\mu_{0}$ in $\eta$ (respectively, of degree $\nu_{0}$ in $\lambda$ ), then after a Weierstrass change of variables among the $\eta_{i}$ only (respectively, the $\lambda_{i}$ only), $f$ will be regular in the usual sense of Definition 3.1 in $\eta_{M}$ (respectively $\lambda_{N}$ ). Similarly, let $f \in U_{m+M, n+N}(\mathbb{Z})$ and suppose that $\sigma(f)$ is regular of degree $\mu_{0}$ in $\eta$ (respectively, of degree $\nu_{0}$ in $\lambda$ ) at the point $(a, b) \in\left(F^{\circ}\right)^{m} \times\left(F^{\circ}\right)^{n}$. Then, after a Weierstrass change of variables among the $\eta_{i}$ only (respectively, the $\lambda_{i}$ only), the power series

$$
a_{\mu_{0}, 0}^{-1}(a, b) \sigma(f)(a, b, \eta, \rho)
$$

(respectively, $\left.a_{0, \nu_{0}}^{-1}(a, b) \sigma(f)\right)$ is regular in $\eta_{M}$ (respectively, $\lambda_{N}$ ). 
(ii) If $f=\sum_{\mu \nu} a_{\mu \nu}(\xi, \rho) \eta^{\mu} \lambda^{\nu}=\sum_{\nu} f_{\nu} \lambda^{\nu}$, where $f_{\nu}=\sum_{\mu} a_{\mu \nu} \eta^{\mu}$, is preregular of degree $\left(\mu_{0}, \nu_{0}\right)$, then $f_{\nu_{0}}$ is regular of degree $\mu_{0}$ in $\eta$.

The following lemma is the analogue for $U_{m, n}$ of [LR4, Lemma 3.1.6] and [LR5. Lemma 4.1], and as in [LR5] plays a key role in the quantifier elimination.

(3.7) Lemma (Bound on degree of preregularity). Let $\xi=\left(\xi_{1}, \ldots, \xi_{m}\right), \eta=$ $\left(\eta_{1}, \ldots, \eta_{M}\right), \rho=\left(\rho_{1}, \ldots, \rho_{n}\right), \lambda=\left(\lambda_{1}, \ldots, \lambda_{N}\right)$ and

$$
f=\sum_{\mu, \nu} f_{\mu \nu}(\xi, \rho) \eta^{\mu} \lambda^{\nu} \in U_{m+M, n+N}(\mathbb{Z}),
$$

where the $f_{\mu \nu}(\xi, \rho) \in U_{m, n}(\mathbb{Z})$. There are $d \in \mathbb{N}$, and $g_{\mu \nu} \in(\rho, \lambda) U_{m+M, n+N}(\mathbb{Z})$, $|(\mu, \nu)|<d$, such that

$$
f=\sum_{|(\mu, \nu)|<d} f_{\mu \nu} \eta^{\mu} \lambda^{\nu}\left(1+g_{\mu \nu}\right) .
$$

Proof. Let $J$ be the ideal of $U_{m, n}(\mathbb{Z})$ generated by the $f_{\mu \nu}$. Since $U_{m, n}(\mathbb{Z})$ is Noetherian, by the Artin-Rees Lemma [Mat, Theorem 8.5], there are $c, d \in \mathbb{N}$ such that

(i) $\left\{f_{\mu \nu}\right\}_{|(\mu, \nu)|<d}$ contains generators for both $J$ and $(\rho)^{c} \cap J$, and

(ii) for any $\alpha \in \mathbb{N}^{m}, \beta \in \mathbb{N}^{n}$ and $e \in \mathbb{N}$, if $f_{\alpha \beta} \in(\rho)^{e+c} U_{m, n}(\mathbb{Z}) \cap J$, then

$$
f_{\alpha \beta}=\sum_{|(\mu, \nu)|<d} h_{\mu \nu} f_{\mu \nu}
$$

for some $h_{\mu \nu} \in(\rho)^{e} U_{m, n}(\mathbb{Z})$.

The following corollary is a consequence of Lemma 3.7.

(3.8) Corollary. Let $f(\xi, \rho, \eta, \lambda)$ be a $D$-function in which $D$ is not applied to any term containing $\eta$ or $\lambda$, so

$$
f=\sum_{\mu, \nu} f_{\mu \nu}(\xi, \rho) \eta^{\mu} \lambda^{\nu}
$$

There is a $d \in \mathbb{N}$ such that for any field $F$ satisfying the axioms of Definition 2.1(ii) and every $(a, b) \in\left(F^{\circ}\right)^{m} \times\left(F^{\circ \circ}\right)^{n}$, if $\sigma(f)(a, b, \eta, \lambda) \not \equiv 0$, then $f$ is preregular at $(a, b)$ of some degree $\left(\mu_{0}, \nu_{0}\right)$ with $\left|\left(\mu_{0}, \nu_{0}\right)\right|<d$. Furthermore, for each such $\left(\mu_{0}, \nu_{0}\right)$ there is a $D$-function $g$ in which $D$ is not applied to any term containing $\eta$ or $\lambda$ such that whenever $f$ is preregular at $(a, b)$ of degree $\left(\mu_{0}, \nu_{0}\right)$,

$$
f(a, b, \eta, \lambda)=f_{\mu_{0} \nu_{0}}(a, b) g(a, b, \eta, \lambda)
$$

for all $(\eta, \lambda) \in\left(F^{\circ}\right)^{M} \times\left(F^{\circ \circ}\right)^{N}$. Furthermore, $g$ is preregular of degree $\left(\mu_{0}, \nu_{0}\right)$. Indeed, the coefficient of $\eta^{\mu_{0}} \lambda^{\nu_{0}}$ is 1 .

Proof. Replacing terms of the form $D\left(t_{1}, t_{2}\right)$ in $f$ by new variables, we may assume that $f \in U_{m+M, n+N}(\mathbb{Z})$. By Lemma 3.7, there is a $d \in \mathbb{N}$ and $g_{\mu \nu} \in$ $(\rho, \lambda) U_{m+M, n+N}(\mathbb{Z}),|(\mu, \nu)|<d$, such that

$$
f=\sum_{|(\mu, \nu)|<d} f_{\mu \nu} \eta^{\mu} \lambda^{\nu}\left(1+g_{\mu \nu}\right) .
$$

The first part of the corollary now follows. 
Now fix $\left(\mu_{0}, \nu_{0}\right)$ with $\left|\left(\mu_{0}, \nu_{0}\right)\right|<d$. Put

$$
\begin{aligned}
g:=\eta^{\mu_{0}} \lambda^{\nu_{0}}\left(1+g_{\mu_{0}, \nu_{0}}\right) & +\sum_{(\mu, \nu) \in I} D_{1}\left(f_{\mu \nu}, f_{\mu_{0} \nu_{0}}\right) \eta^{\mu} \lambda^{\nu}\left(1+g_{\mu \nu}\right) \\
& +\sum_{(\mu, \nu) \in J} D_{0}\left(f_{\mu \nu}, f_{\mu_{0} \nu_{0}}\right) \eta^{\mu} \lambda^{\nu}\left(1+g_{\mu \nu}\right),
\end{aligned}
$$

where

$$
\begin{aligned}
& I:=\left\{(\mu, \nu):|(\mu, \nu)|<d \text { and either } \nu<\nu_{0}, \text { or } \nu=\nu_{0} \text { and } \mu>\mu_{0}\right\}, \text { and } \\
& J:=\left\{(\mu, \nu):|(\mu, \nu)|<d \text { and }\left(\mu_{0}, \nu_{0}\right) \neq(\mu, \nu) \notin I\right\} .
\end{aligned}
$$

To see that $(*)$ is satisfied for all points $(a, b)$ at which $f$ is preregular of degree $\left(\mu_{0}, \nu_{0}\right)$, use Definition 3.5, together with Definition 2.1 and the observation that $I$ and $J$ are finite sets.

(3.9) Remark. The analog of Corollary 3.8 with $S$ in place of $U$ and Definition 5.1 in place of Definition 2.1 also holds. The proof uses [LR5, Lemma 4.1] in place of Lemma 3.7.

\section{UNIFORM RIGID ANALYTIC ELIMINATION}

In this section we prove the Uniform Analytic Elimination Theorem (Theorem 4.2). The proof depends on a double induction, which is isolated in Lemma 4.1. Corollaries 4.3, 4.4 and 4.5 give quantifier simplification, model completeness and the existence of prime models. Corollary 4.6 gives a uniform version of the smooth stratification theorem of [LR7].

Let $\varphi(\xi, \rho, \eta, \lambda)$ be a quantifier-free formula in $L_{a n}^{D}(U)$ or $L_{a n}^{D}(S)$ in which $D$ is not applied to terms involving $\eta, \lambda$. The $(\eta, \lambda)-\operatorname{rank}$ of $\varphi$ is defined to be $(m, n)$, where $m$ is the number of $\eta$ 's (respectively $n$ is the number of $\lambda$ 's) which have non-polynomial occurrence in $\varphi$. We order the set $\mathbb{N} \times \mathbb{N}$ of ranks lexicographically.

The proof of the Uniform Analytic Elimination Theorem 4.2 (see also LR5, Theorem 4.2]), proceeds by induction on rank. The induction step is provided by the following lemma, together with the corresponding algebraic elimination theorem.

(4.1) Lemma. Let $\eta=\left(\eta_{1}, \ldots, \eta_{m}\right), \lambda=\left(\lambda_{1}, \ldots, \lambda_{n}\right), \eta^{*}=\left(\eta_{0}, \eta\right), \lambda^{*}=\left(\lambda_{0}, \lambda\right)$, and let $\varphi(\xi, \rho, \eta, \lambda)$ be a quantifier-free $L_{a n}^{D}(U)$-formula (respectively, an $L_{a n}^{D}(S)$ formula) in which $D$ is not applied to terms involving $\eta, \lambda$. There is a finite set of quantifier-free $L_{a n}^{D}(U)$-formulas (respectively, $L_{a n}^{D}(S)$-formulas) $\psi_{i}\left(\xi, \rho, \eta^{*}, \lambda^{*}\right)$ with $\left(\eta^{*}, \lambda^{*}\right)$-rank $<(m, n)$ and in which $D$ is not applied to terms involving $\eta^{*}, \lambda^{*}$, such that

$$
F^{\circ} \vDash \exists \eta \exists \lambda \varphi \leftrightarrow \bigvee_{i} \exists \eta^{*} \exists \lambda^{*} \psi_{i}
$$

for every field $F$ that satisfies the power series identities of Definition 2.1(ii) (respectively, Definition 5.1(ii)). For example, $F$ can be any complete valued field (respectively, any complete valued field containing the coefficient field $K$ of $S$ ).

Proof. We give the proof for $\varphi$ an $L_{a n}^{D}(U)$-formula. The proof for $\varphi$ an $L_{a n}^{D}(S)$ formula is obtained by replacing the use of Lemma 3.8 by [LR4, Lemma 3.1.6] and Definition 3.5 by [LR4, Definition 2.3.4].

We will apply Corollary 3.8 to each function $f^{(i)}$ that occurs in $\varphi$. Write

$$
f^{(i)}=\sum f_{\mu \nu}^{(i)} \eta^{\mu} \lambda^{\nu}, i=1, \ldots, \ell,
$$


where the $f_{\mu \nu}^{(i)}$ are $D$-functions in the parameters $\xi$ and $\rho$. By Corollary 3.8, there is a $d \in \mathbb{N}$ such that for every field $F$ as in the statement of the lemma, every point $(\bar{\xi}, \bar{\rho}) \in\left(F^{\circ}\right)^{M} \times\left(F^{\circ \circ}\right)^{N}$, and every index $i$, either $\sigma\left(f^{(i)}\right)(\bar{\xi}, \bar{\rho}, \eta, \lambda)$ vanishes identically or there is a $\left|\left(\mu_{i}, \nu_{i}\right)\right|<d$ such that $\sigma\left(f^{(i)}\right)$ is preregular of degree $\left(\mu_{i}, \nu_{i}\right)$ at $(\bar{\xi}, \bar{\rho})$. Splitting into cases by conjoining the respective inequalities of Definition 3.5 (for $|(\mu, \nu)|<d$ ), we may assume that $f^{(i)}$ is preregular of degree $\left(\mu_{i}, \nu_{i}\right)$ at any $(\bar{\xi}, \bar{\rho})$ that satisfies (the modified formula) $\exists \eta \exists \lambda \varphi$. Corollary 3.8 allows us to write $f^{(i)}=f_{\mu_{i} \nu_{i}}^{(i)} g^{(i)}$, where $g^{(i)}$ is preregular of degree $\left(\mu_{i}, \nu_{i}\right)$. Writing

$$
g^{(i)}=\sum g_{\nu}^{(i)} \lambda^{\nu}
$$

as in Remark 3.6(ii), we have $g_{\nu_{i}}^{(i)}$ regular of degree $\mu_{i}$ in $\eta$. It is split further into cases by conjoining one of the two conditions $\left|g_{\nu_{i}}^{(i)}\right|=1$ or $\left|g_{\nu_{i}}^{(i)}\right|<1$ for each $i$.

Case A. A condition $\left|g_{\nu_{i}}^{(i)}\right|<1$ occurs.

Let $\lambda_{0}$ be a new variable of the maximal ideal sort. Conjoin the equation $g_{\nu_{i}}^{(i)}-$ $\lambda_{0}=0$. After a Weierstrass change of variables among the $\eta_{i}$, we may assume that $g_{\nu_{i}}^{(i)}-\lambda_{0}$ is regular in $\eta_{m}$ of degree $s_{i}$. Using Corollary 3.3 and Proposition 3.4, we may replace $g_{\nu_{i}}^{(i)}-\lambda_{0}$ by a Weierstrass polynomial in $\eta_{m}$, and every other function by its remainder on division by $g_{\nu_{i}}^{(i)}-\lambda_{0}$. The resulting formula has rank at most $(m-1, n+1)<(m, n)$.

Case B. The condition $\bigwedge_{i=1}^{\ell}\left|g_{\nu_{i}}^{(i)}\right|=1$ occurs.

We may assume that each occurrence of $f^{(i)}$ is of the form $\left|f^{(i)}\right|$. Because we have conjoined the inequalities of Corollary 3.8, we may replace $\left|f^{(i)}\right|$ by $\left|f_{\mu_{i} \nu_{i}}^{(i)}\right|\left|g^{(i)}\right|$. Since the bound variables $\eta$ and $\lambda$ do not occur in $f_{\mu_{i} \nu_{i}}^{(i)}$, we focus on the occurrence of $\left|g^{(i)}\right|$.

Let $\eta_{0}$ be a new variable of the valuation ring sort. Conjoin the equation

$$
\eta_{0} \prod_{i=1}^{\ell} g_{\nu_{i}}^{(i)}-1=0 .
$$

Since the $g_{\nu_{i}}^{(i)}$ are regular of some degree in $\eta$, so is $g:=\eta_{0} \prod_{i=1}^{\ell} g_{\nu_{i}}^{(i)}-1$. Hence after a change of variables among the $\eta$, by Corollary 3.3 and Proposition 3.4, we may replace $g$ by a Weierstrass polynomial in $\eta_{0}$. Since it is of absolute value 1 , we obtain an equivalent formula by multiplying each $g^{(i)}$ by

$$
\eta_{0} g_{\nu_{1}}^{(1)} \ldots \widehat{g_{\nu_{i}}^{(i)}} \ldots g_{\nu_{\ell}}^{(\ell)}
$$

where the factor marked $\widehat{ }$ is omitted. The coefficient of $\lambda^{\nu_{i}}$ is $\eta_{0} \prod_{i=1}^{\ell} g_{\nu_{i}}^{(i)}$, which by $(*)$, we replace by 1 . We continue to denote the result by $g^{(i)}$. After this process, each $g^{(i)}$ is preregular of degree $\left(0, \nu_{i}\right)$. After a Weierstrass change of variables among the $\lambda$, we may assume that each $g^{(i)}$ is regular in $\lambda_{n}$ of some degree, and hence by Corollary 3.3 and Proposition 3.4, that each $g^{(i)}$ is a Weierstrass polynomial in $\lambda_{n}$. Using Corollary 3.3 and Proposition 3.4 to replace each coefficient of each polynomial $g^{(i)}$ by its remainder upon division by $g$, we obtain an equivalent formula that is polynomial in both $\eta_{0}$ and $\lambda_{n}$, and thus has rank $\leq(m, n-1)<$ $(m, n)$. 
(4.2) Theorem (Uniform Analytic Elimination). Let $\varphi$ be an $L_{a n}^{D}(U)$-formula. Then there is a quantifier-free $L_{a n}^{D}(U)$-formula $\psi$ such that for every valuation ring $F^{\circ}$ that satisfies the axioms of Definition 2.1 (for example, take $F$ algebraically closed and complete in the absolute value $|\cdot|: F \rightarrow \mathbb{R}$ ) we have

$$
F^{\circ} \vDash \varphi \leftrightarrow \psi .
$$

Proof. Let $\varphi(\xi, \rho, \eta, \lambda)$ be a quantifier-free $L_{a n}^{D}(U)$-formula. It suffices to show that the formula $\exists \eta \exists \lambda \varphi$ is equivalent to a quantifier-free $L_{a n}^{D}(U)$-formula. Since the relations $D_{0}(x, y)=z$ and $D_{1}(x, y)=z$ are defined uniformly for all valued fields by a quantifier-free $L$-formula, at the expense of more existential quantifiers we may assume that $D$ is not applied to any term involving $\eta$ or $\lambda$ in $\varphi$, which is the condition of Lemma 4.1. We now proceed by induction on the rank of $\varphi$, using the Algebraic Elimination Theorem [W] to eliminate existentially quantified polynomially-occurring variables. Note that Lemma 4.1 and the Algebraic Elimination Theorem are independent of the valuation ring $F^{\circ}$.

Since the functions $D_{0}$ and $D_{1}$ are uniformly quantifier-free definable, Theorem 4.2 yields the following.

(4.3) Corollary (Uniform Quantifier Simplification). Let $\varphi$ be an $L_{a n}(U)$-formula. There is an existential $L_{a n}(U)$-formula $\psi$ such that in every valuation ring $F^{\circ}$ that satisfies the axioms of Definition 2.1,

$$
F^{\circ} \vDash \varphi \leftrightarrow \psi .
$$

(4.4) Corollary (Model-Completeness). $\mathcal{T}_{a n}(U)$ is model-complete. In particular, let $\varphi\left(x_{1}, \ldots, x_{n}\right)$ be an $L_{a n}^{D}(U)$-formula, let $F_{1}^{\circ} \subset F_{2}^{\circ}$ be two models of $\mathcal{T}_{a n}(U)$ and let $a_{1}, \ldots, a_{n} \in F_{1}^{\circ} \cup F_{1}^{\circ \circ} \cup\left|F_{1}^{\circ}\right|$. Then

$$
F_{1}^{\circ} \vDash \varphi\left(a_{1}, \ldots, a_{n}\right) \leftrightarrow F_{2}^{\circ} \vDash \varphi\left(a_{1}, \ldots, a_{n}\right) .
$$

The theory $\mathcal{T}_{a n}(U)$ is the uniform theory of rigid subanalytic sets; more precisely:

(4.5) Corollary. $\quad$ (i) Let $F$ be a complete valued field. Then

$$
F_{\text {alg }}^{\circ} \vDash \mathcal{T}_{\text {an }}(U) .
$$

(ii) For each prime $p$, the theories

$$
\mathcal{T}_{\text {an }}(U) \cup\{\text { characteristic is } p\},
$$

$\mathcal{T}_{\text {an }}(U) \cup\{$ characteristic is 0 and residue characteristic is $p\}$,

$$
\mathcal{T}_{\text {an }}(U) \cup\{\text { residue characteristic is } 0\}
$$

have prime models, namely

$$
\left(\mathbb{F}_{p}((t))\right)_{\text {alg }}^{\circ}, \quad\left(\mathbb{Q}_{p}\right)_{\text {alg }}^{\circ} \text { and }(\operatorname{Frac} \mathbb{Z}[[t]])_{\text {alg }}^{\circ},
$$

respectively, where Frac denotes the field of fractions. Hence each of these theories is complete.

(iii) Any valuation ring can be extended to a model of $\mathcal{T}_{\text {an }}(U)$. In particular, the valuation ring of any maximally complete, algebraically closed, valued field is a model of $\mathcal{T}_{\text {an }}(U)$. 
Proof. Part (i) is immediate from Theorem 4.2.

For the equicharacteristic $p$ case of part (ii), let $F^{\circ}$ be a model of $\mathcal{T}_{a n}(U)$ of equicharacteristic $p$. Since $F^{\circ}$ is non-trivially valued, there must be some $t \in F^{\circ}$ with $0<|t|<1$. Consider the map

$$
\varepsilon: U_{0,1} \rightarrow F^{\circ}: f \mapsto \sigma(f)(t) .
$$

The identities of Definition 2.1(ii) guarantee that $\varepsilon$ is a homomorphism. Let $f \in$ $U_{0,1}$. By Weierstrass Preparation (Corollary 3.3), there is a unit $u$ of $U_{0,1}$ and a polynomial $P \in \mathbb{Z}[\rho]$ such that $f=u \cdot P$. Thus $f \in$ ker $\varepsilon$ if, and only if, $\varepsilon(P)=0$. Since $t$ is transcendental over $\mathbb{F}_{p}, \operatorname{ker} \varepsilon=(p)$. Therefore, $F^{\circ}$ contains an isomorphic copy of the valuation ring of the complete field $\mathbb{F}_{p}((t))$. Moreover, since Definition 2.1(i) guarantees that $F$ is algebraically closed, $F^{\circ}$ contains $\mathbb{F}_{p}((t))_{\text {alg }}^{\circ}$, which by part (i) is a model of $\mathcal{T}_{a n}(U)$. The completeness of the theory $\mathcal{T}_{a n}(U) \cup$ $\{$ characteristic is $p\}$ is now a consequence of Corollary 4.4. The equicharacteristic zero and mixed characteristic cases are similar.

Part (iii) is an immediate consequence of Theorem 5.9, below.

Let $\varphi\left(\xi_{1}, \ldots, \xi_{m}\right)$ be an $L_{a n}^{D}(U)$-formula. For each algebraically closed complete ultrametric field $F$ let $X_{\varphi}(F)$ be the subanalytic set in $\left(F^{\circ}\right)^{m}$ defined by $\varphi$; i.e.,

$$
X_{\varphi}(F):=\left\{\left(x_{1}, \ldots, x_{m}\right) \in\left(F^{\circ}\right)^{m}: \varphi\left(x_{1}, \ldots, x_{m}\right)\right\} .
$$

By [LR7] there is a stratification of $X_{\varphi}(F)$ into a finite union of disjoint subanalytic manifolds, defined in $L_{a n}^{D}\left(\bigcup_{m, n} S_{m, n}(E, K)\right)$. The results of [LR5] show that the same stratification is valid for all $F^{\prime} \supset K, F^{\prime}$ algebraically closed and complete. An examination of the proof of [LR7] shows that the stratification (for $X_{\phi}(F)$ ) is actually defined over $L_{a n}^{D}(U)$.

(4.6) Corollary (Uniform Smooth Stratification). Let $\varphi$ be an $L_{a n}^{D}(U)$-formula. Then there are $L_{a n}^{D}(U)$-formulas $\psi^{0}, \ldots, \psi^{d}$ with the following properties. For every valuation ring $F^{\circ}$ that satisfies the axioms of Definition 2.1,

(i) $X_{\varphi}(F)=\bigcup_{i=0}^{d} X_{\psi^{i}}(F)$,

(ii) $X_{\psi^{i}}(F) \cap X_{\psi^{j}}(F)=\emptyset$ if $i \neq j$ and

(iii) $X_{\psi^{i}}(F)$ is either $\emptyset$ or an $F$-analytic manifold of dimension $i, i=0, \ldots, d$.

(We say that $\psi=\bigvee_{i=0}^{d} \psi^{i}$ defines a smooth stratification of $X_{\varphi}$.)

Proof. First consider a model $F^{\circ}$ of $\mathcal{T}_{a n}(U)$ with Char $\widetilde{F}=0$. Let $\psi_{0}=\bigvee_{i=0}^{d} \psi_{0}^{i}$ be an $L_{a n}^{D}(U)$-formula defining a smooth stratification of $X_{\varphi}(F)$. By Corollary 4.5, $\psi_{0}$ defines a smooth stratification of $X_{\varphi}\left(F^{\prime}\right)$ for every algebraically closed, complete ultrametric field $F^{\prime}$ of equicharacteristic zero. An application of Theorem 4.2 to the formula " $\psi_{0}$ gives a smooth stratification of the set defined by $\varphi$ ", where smoothness is detected by the non-vanishing of a certain Jacobian, yields a variable-free sentence $\chi$ in $L_{a n}^{D}$ which is true in equicharacteristic zero, and hence in residue characteristic $\geq e$. Let $\psi_{p}=\bigvee_{i=0}^{d} \psi_{p}^{i}$ and $\theta_{p}=\bigvee_{i=0}^{d} \theta_{p}^{i}$ define smooth stratifications, respectively, of $X_{\varphi}(F)$, where Char $F=0$ and Char $\widetilde{F}=p$ (respectively, Char $F=p$ ) for $p<e$. Now, for $i=1, \ldots, d$, let $\psi^{i}$ be the formula

$$
\left(|e !|=1 \wedge \psi_{0}^{i}\right) \vee \bigvee_{p<e}\left(0 \neq|p|<1 \wedge \psi_{p}^{i}\right) \vee \bigvee_{p<e}\left(p=0 \wedge \theta_{p}^{i}\right)
$$


(4.7) Remark. A one-dimensional subanalytic set is semi-algebraic (see [LR1]) and hence is a finite Boolean combination of discs. For each value $\bar{x}$ of the parameters $x$, an $L_{a n}^{D}(S(K))$-formula $\varphi\left(x_{1}, \ldots, x_{n}, t\right)$ defines a subanalytic set in the "line" $F^{\circ}$. It was shown in LR3] that there is a bound $b$, independent of the value of the parameter $x$, on the minimum number of discs needed to describe the set $\varphi(\bar{x}, t)$ as a Boolean combination of these discs. By model-completeness, the bound is uniform in all models of the theory $\mathcal{T}_{a n}(K)$ described in Definition 5.1, below. If $\varphi$ is an $L_{a n}^{D}(U)$-formula, then the bound depends only on the pair $\chi=(p, q)$ of characteristics of the fields $K, \widetilde{K}$. It follows from the Uniform Rigid Analytic Elimination Theorem 4.2 that there is, in fact, a uniform bound $b$ valid for all models $F$ of $\mathcal{T}_{a n}(U)$ of any characteristic $(p, q)$ and all values $\bar{x}$ of the parameters $x$. (See also $\mathrm{vHM}$ for a $p$-adic analogue.)

(4.8) Remark (Uniform Analytic Continuation). One consequence of the quasiaffinoid Acyclicity Theorem of [LR6] is that if $U_{0}, \ldots, U_{N}$ is an admissible [LR6] Definition 2.3.1] cover of the polydisc $\left(F^{\circ}\right)^{m} \times\left(F^{\circ 0}\right)^{n}$ and if $f_{i} \in \mathcal{O}\left(U_{i}\right)$ (i.e., the $f_{i}$ are quasi-affinoid functions on $\left.U_{i}\right)$ such that the restrictions $\left.f_{i}\right|_{U_{i} \cap U_{j}}=\left.f_{j}\right|_{U_{i} \cap U_{j}}$, then there is an $f \in S_{m, n}$ such that $\left.f\right|_{U_{i}}=f_{i}$. In particular, in characteristic zero, if $0 \in U_{0}$, the Taylor series expansion of $f_{0}$ is $f$. The Uniform Rigid Analytic Elimination Theorem 4.2 yields a uniform version of this, as follows. Suppose that the $L_{a n}^{D}(U)$-formulas $\psi_{0}, \ldots, \psi_{N}$ define an admissible cover $U_{0}, \ldots, U_{N}$ of $\left(F^{\circ}\right)^{m} \times$ $\left(F^{\circ \circ}\right)^{n}$, where $F$ is an equicharacteristic 0 model of the theory $\mathcal{T}_{a n}(U)$. (It is equivalent to assume that $\left\{U_{0}, \ldots, U_{N}\right\}$ is an admissible cover of $\left(F^{\circ}\right)^{m} \times\left(F^{\circ \circ}\right)^{n}$ for models $F$ of $\mathcal{T}_{a n}(U)$ of all but finitely many characteristics $\chi=(p, q)$.) Let $t_{0}, \ldots, t_{N}$ be terms of $L_{a n}^{D}(U)$ such that each $\left.t_{i}\right|_{U_{i}} \in \mathcal{O}\left(U_{i}\right)$ and $\left.t_{i}\right|_{U_{i} \cap U_{j}}=\left.t_{j}\right|_{U_{i} \cap U_{j}}, i, j=$ $0, \ldots, N$. Note that this can all be expressed by an $L_{a n}^{D}(U)$-formula. The Acyclicity Theorem of [LR6] guarantees that there is an $f \in S_{m, n}(\mathbb{Q})$ such that $\left.f\right|_{U_{i}}=t_{i}, i=$ $0, \ldots, N$. Assume $0 \in U_{0}$. Comparing partial derivatives of $t_{0}$ at 0 with partial derivatives of $f$ at 0 , using the Chain Rule and Quotient Rule, and the fact that $t_{0}$ is an analytic $D$-function on $U_{0}$, we see that all coefficients of $f$ are in $\mathbb{Z}[1 / \ell]$ for some $\ell \neq 0$, so $f \in U_{m, n}(\mathbb{Q})$. Applying the Uniform Rigid Analytic Elimination Theorem 4.2 to the $L_{a n}^{D}(U)$-formulas $\left.f\right|_{U_{i}}=t_{i}$ yields the desired conclusion for all but finitely many characteristics $\chi=(p, q)$. The same is true for admissible covers of uniformly defined varieties.

\section{AXIOMS AND NON-STANDARD MODELS OF THE ANALYTIC THEORY}

It is clear from the proofs of the quantifier elimination and simplification theorems of [LR5] that those theorems hold for the valuation ring $F^{\circ}$ of any algebraically closed valued field $F$ extending the coefficient field $K$ on which the functions in $S(E, K)$ (or $\mathcal{E}(T)$ or $\mathcal{E}(\mathcal{H})$; see [LR5]) "make sense" and satisfy the identities true to these functions in the rings $S_{m, n}(E, K)$. We summarize the statements of those theorems below, and make our assertion concerning non-standard models of the analytic theory more precise in Definition 5.1 and Theorem 5.2 and in Definition 5.4 and Theorem 5.9, where we show that any model can be extended to one that is maximally complete. The results of this section are useful for the treatment of the Lojasiewicz inequalities given in Section 6.

We showed in [LR5] that if $S=\bigcup_{m, n} S_{m, n}(E, K)$, if $K \subset F$ is any complete valued field extending $K$ and if $F_{\text {alg }}$ is the algebraic closure of $F$, then $F_{a l g}^{\circ}$ admits 
quantifier elimination in the language $L_{a n}^{D}(S)$. (The language $L_{a n}^{D}(S)$ is the language $L$ enriched with $D_{0}, D_{1}$ and symbols for the elements of $S^{\circ}:=\bigcup_{m, n} S_{m, n}^{\circ}$.) We further obtained quantifier elimination theorems for many sublanguages of $L_{a n}^{D}(S)$. The most interesting example is that $F_{\text {alg }}^{\circ}$ admits elimination of quantifiers in the language $L_{a n}\left(\mathcal{E}(T)\right.$ ), where $T=\bigcup_{m} T_{m}(K)$ and $\mathcal{E}(T)$ is (roughly speaking) the set of analytic functions given by $L_{a n}^{D}(S)$-terms which are existentially definable over the ring of strictly convergent power series $T$. This yields a quantifier simplification theorem in the language $L_{a n}(T)$. These quantifier elimination and quantifier simplification theorems were shown to be uniform in $F_{\text {alg }}^{\circ}$, for all complete, valued field extensions $F$ of the coefficient field $K$ (see [LR5], Theorem 4.2, Corollary 4.4 and Corollary 4.5). In this section, we extend that result to a larger class of field extensions $F$ of $K$. For technical reasons, we restrict the class of separated power series rings $S_{m, n}(E, K)$ to those where $E$ is a discrete valuation ring.

(5.1) Definition. Let $S:=\bigcup_{m, n} S_{m, n}^{\circ}(E, K)$, where $E \subset K^{\circ}$ is a discrete valuation ring. The axioms $\mathcal{T}_{a n}(K)$ in the language $L_{a n}(S)$ for the analytic theory of $K_{a l g}$ are:

(i) the axioms, in the 3-sorted language $L$, for the valuation ring of an algebraically closed, non-Archimedean, non-trivially valued field containing $K$, and

(ii) all identities of the form

$$
f=F\left(g_{1}, \ldots, g_{m}, h_{1}, \ldots, h_{n}\right),
$$

where $F \in S_{m, n}^{\circ}, g_{1}, \ldots, g_{m} \in S_{M, N}^{\circ}$ and $h_{1}, \ldots, h_{n} \in(\rho) \cdot S_{M, N}^{\circ}+S_{M, N}^{\circ \circ}$. Note that this includes all Weierstrass data and the diagram of the ring $S^{\circ}=\bigcup_{m, n} S_{m, n}^{\circ}$.

The axioms $\mathcal{T}_{a n}^{D}(K)$ in $L_{\text {an }}^{D}$ are those of $\mathcal{T}_{a n}(K)$ together with the obvious axioms defining $D_{0}$ and $D_{1}$.

The theories in the various sublanguages mentioned at the beginning of this section are obtained by restriction.

The proofs in [LR5] only use facts about $S$ and the various subsets, such as the strong Noetherian property, the Weierstrass Division Theorem, the algebraic elimination theorem, etc., all of which are coded into $\mathcal{T}_{\text {an }}$ and $\mathcal{T}_{\text {an }}^{D}$. Hence we have the following.

(5.2) Theorem. The theories $\mathcal{T}_{a n}(K)$ of Definition 5.1 admit quantifier simplification in $L_{a n}$ and quantifier elimination in $L_{a n}^{D}$.

The following simple example will be useful in Section 6 .

(5.3) Remark. An obvious example of a model of $\mathcal{T}_{a n}(K)$ which is not complete (and not the algebraic closure of a complete field) is $F:=\left(K_{a l g}\right)^{*}$, a nonprincipal ultrapower of the algebraic closure of $K$. A more interesting class of examples is given by the following. Let $F$ be any model of $\mathcal{T}_{a n}(K)$, e.g., $F=\left(K_{a l g}\right)^{*}$. Thus $F$ is a valued field extending $K_{\text {alg }}$. Let $I \subset F^{\circ}$ be a prime ideal with $I \cap K^{\circ}=(0)$. Let $F_{1}$ be the quotient field of $F^{\circ} / I$. In the example $F=\left(K_{a l g}\right)^{*}$ we could take $I$ to be the set of all infinitesimals of $F$. We then have that $F_{1}$ is a model of $\mathcal{T}_{\text {an }}(K)$. This is clear since all the functions in $S(K)$ are uniformly continuous. Indeed, let $f \in S_{m, n}(K)$ and $x, y \in\left(F^{\circ}\right)^{m} \times\left(F^{\circ \circ}\right)^{n}$, and suppose $x_{i}-y_{i} \in I$ for 
all $i=1, \ldots, m+n$. Since $|f(x)-f(y)| \leq\|f\||x-y|$, and since for any ideal $I$ of a valuation ring, $a \in I$ and $|b| \leq|a|$ implies $b \in I$, it follows that we can define $f(x+I):=f(x)+I$. Note, however, that $D(x+I, y+I)$ is not in general contained in $D(x, y)+I$. For example, take $y \in I$ and $x:=y+y$. But since $F_{1}$ is a valuation ring, there are functions $D_{0}$ and $D_{1}$ on $F_{1}$ that satisfy the axioms.

(5.4) Definition. A valued field $F$ is maximally complete iff there is no proper extension $F \subsetneq F_{1}$ with $\tilde{F}_{1}=\tilde{F}$ and $\left|F_{1}\right|=|F|$.

Let $K$ be a valued field. For a given algebraically closed residue field extension $k \supset \widetilde{K}$ and ordered divisible group $G \supset|K|$, there is a unique maximally complete field $F$ containing $K$ such that $\widetilde{F}=k$ and $|F|=G$. See [Kap1, Kap2 and [ $\mathrm{Po}$. Such a field is algebraically closed.

Our next goal is to show that the functions of $S_{m, n}^{\circ}(E, K)$ extend to functions on any maximally complete $F \supset K$, so that $F^{\circ}$ becomes a model of $\mathcal{T}_{a n}(K)$. Recall that $E \subset K^{\circ}$ is a discrete valuation ring.

Fix a maximally complete algebraically closed extension field $F \supset K$ with (algebraically closed) residue field $\widetilde{F} \supset \widetilde{K}$ and (ordered divisible) value group $G=|F| \supset|K|$. Following Poonen [Po, Sections 4 and 5], let $E^{\prime}$ be a discrete valuation ring with residue field $\widetilde{F}$; without loss of generality, we can assume that $E^{\prime} \supset E$, the discrete valuation ring used in the above definition of $S_{m, n}(E, K)$. Consider the Mal'cev-Neumann ring $E^{\prime}((G))$ of formal sums $\sum_{g \in G} e_{g} \cdot g$, where the set $\left\{g \in G: e_{g} \neq 0\right\}$ is reverse well ordered (we have to reverse the given order on $G$ because we write our valuations multiplicatively) by $[\mathrm{Po}], F=E^{\prime}((G)) / N$, where $N$ is the maximal ideal of null series. Let $\mathcal{S}$ be a set of representatives of equivalence classes of $E^{\prime}$ modulo its maximal ideal. By [ $\mathrm{Po}$, Proposition 4.4], any element $a \in F$ has a unique representation in $E^{\prime}((G))$ of the form $\beta=\sum_{g \in G} \beta_{g} \cdot g$, with $\beta_{g} \in \mathcal{S}$. We define

$$
\operatorname{Supp}(a):=\left\{g \in G: \beta_{g} \neq 0\right\}
$$

note that $\operatorname{Supp}(a)$ is reverse well ordered because $\beta \in E^{\prime}((G))$.

Let $B \in \mathcal{B}$ (in the definition of $S_{m, n}(E, K)$ ), so $B \subset K^{\circ}$ and

$$
B=E\left[a_{i}: i \in \mathbb{N}\right]_{\left\{a \in E\left[a_{i}: i \in \mathbb{N}\right]:|a|=1\right\}}^{\wedge}
$$

where $\left\{a_{i}\right\}$ is a null sequence of $K^{\circ}$.

(5.5) Lemma. $\operatorname{Supp}(B):=\bigcup_{a \in B} \operatorname{Supp}(a)$ is reverse well ordered.

Proof. It is no loss of generality to assume that $\widetilde{E}=\widetilde{K}$ and that $E \subset E^{\prime}$. We have

$$
K \subset E\left(\left(\left|K^{*}\right|\right)\right) /\left(N \cap E\left(\left(\left|K^{*}\right|\right)\right)\right) \subset E^{\prime}((G)) / N
$$

and, without loss of generality, we may assume that $\mathcal{S}$ is chosen so that $(\mathcal{S} \cap K)^{\sim}=\widetilde{K}$. Thus, since $\left|K^{*}\right| \subset \mathbb{R}_{+}$, Supp $K \subset \mathbb{R}_{+}$. By [Pass. Lemmas 13.2 .9 and 13.2.10],

$$
\operatorname{Supp}\left(E\left[a_{i}: i=1, \ldots, n\right]\right)=: A_{n}
$$

is reverse well ordered, and $A_{n+1} \backslash A_{n} \subset\left[0, \varepsilon_{n}\right] \subset \mathbb{R}$ for some sequence $\left\{\varepsilon_{n}\right\}$ with $\varepsilon_{n} \rightarrow 0$. It follows that

$$
\bigcup_{n} \operatorname{Supp}\left(E\left[a_{i}: i=1, \ldots, n\right]\right)=\operatorname{Supp}\left(E\left[a_{i}: i \in \mathbb{N}\right]\right)
$$


is reverse well ordered. For any $\varepsilon>0$,

$$
\operatorname{Supp}\left(E\left[a_{i}: i \in \mathbb{N}\right]^{\wedge}\right) \cap[\varepsilon, 1]=\operatorname{Supp}\left(E\left[a_{i}: i \in \mathbb{N}\right]\right) \cap[\varepsilon, 1]
$$

and hence

$$
\operatorname{Supp}\left(E\left[a_{i}: i \in \mathbb{N}\right]^{\wedge}\right)=\operatorname{Supp}\left(E\left[a_{i}: i \in \mathbb{N}\right]\right),
$$

and so is reverse well ordered. Finally, observe that

$$
\begin{aligned}
\operatorname{Supp}(B) & =\operatorname{Supp}\left(E\left[a_{i}: i \in \mathbb{N}\right]_{\left\{a \in E\left[a_{i}: i \in \mathbb{N}\right]:|a|=1\right\}}\right) \\
& =\operatorname{Supp}\left(E\left[a_{i}: i \in \mathbb{N}\right]^{\wedge}\right)
\end{aligned}
$$

because $\widetilde{E}=\widetilde{K}$, and hence is reverse well ordered.

(5.6) Lemma. Let $\bar{\xi}_{1}, \ldots, \bar{\xi}_{m} \in F^{\circ}, \bar{\rho}_{1}, \ldots, \bar{\rho}_{n} \in F^{\circ \circ}$ and let

$$
A=\left[\operatorname{Supp}(B) \cup \bigcup_{i=1}^{m} \operatorname{Supp}\left(\bar{\xi}_{i}\right) \cup \bigcup_{j=1}^{n} \operatorname{Supp}\left(\bar{\rho}_{j}\right)\right] \backslash\{1\} .
$$

Then $\widetilde{A}:=\bigcup_{n \in \mathbb{N}} A^{n}$ is well ordered and $\bigcap_{n \in \mathbb{N}} \widetilde{A}^{n}=\emptyset$.

Proof. Pass, Lemma 13.2.10.

(5.7) Lemma. Let $f=\sum a_{\mu \nu} \xi^{\mu} \rho^{\nu} \in B\langle\xi\rangle[[\rho]]$ and let $\bar{\xi} \in\left(F^{\circ}\right)^{m}, \bar{\rho} \in\left(F^{\circ \circ}\right)^{n}$. Then the sum $f(\bar{\xi}, \bar{\rho})=\sum a_{\mu \nu} \bar{\xi}^{\mu} \bar{\rho}^{\nu}=\sum_{g \in G} e_{g} \cdot g$ is well defined in F; i.e., only finitely many terms contribute to the coefficient of each $g$, and the support of the sum is well ordered. Furthermore, all the identities of Definition 5.1(ii) that hold in $S_{m, n}(E, K)$ also hold among these functions on $\left(F^{\circ}\right)^{m} \times\left(F^{\circ 0}\right)^{n}$.

Proof. Keep the notation from the previous lemma. There are $a_{1}, \ldots, a_{m} \in \mathcal{S} \subset E^{\prime}$ such that $\operatorname{Supp}\left(\tau_{i}\right) \subset A$, where $\tau_{i}:=\bar{\xi}_{i}-a_{i}, i=1, \ldots, m$. Note that $\operatorname{Supp}(B[a])=$ $\operatorname{Supp}(B)$ is reverse well ordered so for any $h \in B\langle\xi\rangle, h(a)$ is well defined and $\operatorname{Supp}(h(a)) \subset \operatorname{Supp}(B)$. Let $\lambda_{1}, \ldots, \lambda_{m}$ be variables and put

$$
\begin{aligned}
g(\lambda, \rho) & :=f(a+\lambda, \rho) \in B\langle a\rangle[[\lambda, \rho]], \\
\bar{\lambda} & :=\bar{\xi}-a .
\end{aligned}
$$

Note that $\operatorname{Supp}(\mu) \subset A$ for each monomial $\mu$ of $g(\bar{\lambda}, \bar{\rho})$ of positive degree. Thus, by Lemma $5.6, g(\bar{\lambda}, \bar{\rho})=f(\bar{\xi}, \bar{\rho})$ is well defined.

(5.8) Proposition. Let $F$ be a (non-trivially) valued field such that $F^{\circ}$ is an $L_{a n}(K)$-structure (respectively, $L_{a n}(U)$-structure) satisfying the power series identities of Definition 5.1(ii) (respectively, Definition 2.1(ii)). Then $F^{\circ}$ is a Henselian valuation ring.

Proof. It suffices to show that any polynomial of the form $P(X):=\sum_{i=0}^{d} a_{i} X^{i} \in$ $F^{\circ}[X]$, with $a_{0} \in F^{\circ \circ}$ and $a_{1}=1$, has a zero in $F^{\circ \circ}$. Put

$$
f(\eta ; \lambda, \rho):=\lambda+\rho+\eta_{2} \rho^{2}+\cdots+\eta_{d} \rho^{d} \in U_{d, 2}(\mathbb{Z}) \subset S_{d, 2} .
$$

Observe that $f$ is regular in $\rho$ of degree 1 , so there is a unit $v$ of $U_{d, 2}$ and an element $g(\eta, \lambda) \in(\lambda) \cdot U_{d, 1}$ such that $f=v \cdot(\rho-g)$. By the power series identities

$$
P\left(g\left(a^{\prime}, a_{0}\right)\right)=v\left(a^{\prime}, a_{0}, g\left(a^{\prime}, a_{0}\right)\right)\left(g\left(a^{\prime}, a_{0}\right)-g\left(a^{\prime}, a_{0}\right)\right)=0,
$$

and since $g \in(\lambda)$, we have that $g\left(a^{\prime}, a_{0}\right) \in F^{\circ 0}$. 
(5.9) Theorem. Let $K$ be a complete, non-Archimedean valued field.

(i) Let $F$ be a maximally complete, algebraically closed, valued field extension of $K$. Then $F$ can be expanded to a model of $\mathcal{T}_{\text {an }}(K)$.

(ii) Let $F^{\circ}$ be as in the statement of Proposition 5.8. Then $F^{\circ}$ can be extended to a model of $\mathcal{T}_{a n}(K)$ (respectively, of $\mathcal{T}_{a n}(U)$ ).

Proof. (i) This is immediate from Lemma 5.7.

(ii) Let $F_{\text {alg }}$ be the algebraic closure of $F$. Since $F^{\circ}$ is Henselian, $F_{\text {alg }}^{\circ}$ is integral over $F^{\circ}$, so $F_{a l g}^{\circ}$ is the direct union of its subextensions $A$ which are finite $F^{\circ}$ modules. The general case is similar to the following example. Take $A=F^{\circ}[\alpha]$ with $|\alpha|<1$. Then $\alpha$ satisfies a polynomial equation $\lambda^{d}+a_{1} \lambda^{d-1}+\cdots+a_{d}$, where $a_{1}, \ldots, a_{d} \in F^{\circ \circ}$. Let $f(\xi, \rho, \lambda) \in S_{m, n+1}$ and fix $\bar{\xi} \in\left(F^{\circ}\right)^{m}$ and $\bar{\rho} \in\left(F^{\circ \circ}\right)^{n}$; we must define $f(\bar{\xi}, \bar{\rho}, \alpha)$. Put $g:=\lambda^{d}+\tau_{1} \lambda^{d-1}+\cdots+\tau_{d}$. By Weierstrass Division (all such divisions are coded in axiom group (ii)),

$$
f=q \cdot g+\sum_{i=0}^{d-1} r_{i}(\xi, \rho, \tau) \lambda^{i}
$$

for some $q \in S_{m, n+d+1}$ and $r_{i} \in S_{m, n+d}$. Now we may define $f(\bar{\xi}, \bar{\rho}, \alpha)$ to be $\sum_{i=0}^{d-1} r_{i}(\bar{\xi}, \bar{\rho}, a) \alpha^{i}$.

\section{6. ŁOJASIEWICZ INEQUALITIES}

In this section, we give an improved treatment of the Lojasiewicz inequalities proved in $[\mathrm{L}$, and show that these inequalities are uniform in various ways.

By analytic set, we mean the zero set of finitely many functions, analytic in an appropriate open domain. There are several rings of analytic functions under consideration for which the analogues of the following lemma are valid. However, we only need the lemma for the case of analytic sets defined over $S_{m, n}$.

(6.1) Lemma. The closure of a Boolean combination $X$ of analytic sets is an analytic set.

Proof. By the Noetherianness of $S_{m, n}$, we reduce to the case that $X=Y \backslash Z$, where $Y$ and $Z$ are analytic sets and $Y$ is irreducible. We may suppose $Z \not \supset Y$; then it suffices to show that $Z$ contains no non-empty open set of $Y$. Suppose not; i.e., suppose $Y \cap U \subset Z$ for some non-empty polydisc $U \subset \operatorname{Max} S_{m, n}$.

There is a finitely generated ideal $I$ such that $Z=V(I)$ and a prime ideal $\mathfrak{p}$ such that $Y=V(\mathfrak{p})$. Let $x \in U$ and let $\mathfrak{m}$ (respectively, $\mathfrak{M}$ ) be the maximal ideal of $S_{m, n}$ (respectively, $\mathcal{O}(U)$ ) corresponding to $x$. Since $V(\mathfrak{p}) \cap U \subset V(I)$, and since $I$ is finitely generated, the Nullstellensatz [LR4, Theorem 4.1.1] yields $I^{\ell} \subset \mathfrak{p} \cdot \mathcal{O}(U)$ for some $\ell \in \mathbb{N}$. Since the natural map $\left(S_{m, n}\right)_{\mathfrak{m}} \rightarrow \mathcal{O}(U)_{\mathfrak{M}}$ is faithfully flat (LR4] Lemma 4.2.8(i)] and its proof), we have $I^{\ell} \subset \mathfrak{p}\left(S_{m, n}\right)_{\mathfrak{m}}$. In other words, since $I$ is finitely generated, $g \cdot I^{\ell} \subset \mathfrak{p}$ for some $g \notin \mathfrak{m} \supset \mathfrak{p}$. Since $\mathfrak{p}$ is prime, we have $I \subset \mathfrak{p}$, contradicting $Z \not \supset Y$.

(6.2) Lemma. Let $X$ be closed and subanalytic; then $X$ is the projection of an analytic set.

Proof. By [LR5, Corollary 5.3], $X$ is the image under a coordinate projection of a set $X^{\prime}$ defined by a quantifier-free $L_{a n}$-formula. Adding new variables $\eta$ (respectively, $\lambda$ ) for each of its atomic subformulas $\left|t_{1}\right| \leq\left|t_{2}\right|$ (respectively, $\left|t_{1}\right|<\left|t_{2}\right|$ ), and 
replacing each such subformula by $t_{1}-\eta t_{2}=0$ (respectively, $t_{1}-\lambda t_{2}=0 \wedge t_{2} \neq 0$ ), we may assume that $X^{\prime}$ is a Boolean combination of analytic sets. Since $X$ is closed and coordinate projection is continuous, we may replace $X^{\prime}$ by its closure, which is an analytic set by Lemma 6.1 .

(6.3) Lemma. Let $X \subset\left(F^{\circ}\right)^{m}$ be a closed subanalytic set. Suppose that for every $\varepsilon \in F^{\circ} \backslash\{0\}$ there is a $\left(\xi_{1}, \ldots, \xi_{m}\right) \in X$ with $\left|\xi_{m}\right|<|\varepsilon|$. Then there are $\xi_{1}, \ldots, \xi_{m-1}$ such that $\left(\xi_{1}, \ldots, \xi_{m-1}, 0\right) \in X$. In particular, if $f$ is an $L_{a n}^{D}$-definable, continuous function with closed domain, then taking $X$ to be its graph, we see that $0=\inf |f(x)|$ implies that $f\left(x_{0}\right)=0$ for some $x_{0}$.

Proof. By Lemma 6.2, it suffices to consider only the case that $X$ is an analytic set, say $X=V\left(f_{1}, \ldots, f_{n}\right)$. Let $F^{*}$ be a non-principal ultrapower of $F$, and let

$$
I=\left\{x \in F^{*}:|x|<|\varepsilon| \text { for all } \varepsilon \in F \backslash\{0\}\right\}
$$

be the ideal of infinitesimals of $\left(F^{\circ}\right)^{*}=\left(F^{*}\right)^{\circ}$. By hypothesis, there are elements $\xi_{1}, \ldots, \xi_{m} \in\left(F^{\circ}\right)^{*}$ such that $f_{i}(\xi)=0,1 \leq i \leq n$, and $\xi_{m} \in I$. Let $\bar{F}$ be the fraction field of $\left(F^{\circ}\right)^{*} / I$; by Remark $5.3, \bar{F}$ is a model of $\mathcal{T}_{a n}(F)$. Since $f_{i}(\xi+I) \subset$ $f_{i}(\xi)+I, 1 \leq i \leq n$, we have $f_{i}\left(\bar{\xi}_{1}, \ldots, \bar{\xi}_{m-1}, 0\right)=0,1 \leq i \leq n$, where $\bar{\xi}_{j}:=\xi_{j}+I$. The lemma follows by Theorem 5.2 .

Let $F^{\circ}$ be a model of $\mathcal{T}_{a n}(S(K))$, suppose that $f_{F}$ is a (partial) function on $\left(F^{\circ}\right)^{n}$ defined by an $L_{a n}(S(K))$-formula $\varphi$, and that $X_{F}$ is a subset of $\left(F^{\circ}\right)^{n}$ defined by an $L_{a n}(S(K))$-formula $\psi$. Then by model-completeness and the existence of a prime model of $\mathcal{T}_{a n}(S(K))$ (Corollary 4.3), $\varphi$ defines a function $f_{F^{\prime}}$ and $\psi$ defines a set $X_{F^{\prime}}$ in each model $F^{\prime}$ of $\mathcal{T}_{a n}(S(K))$, and $f_{F^{\prime}}$ and $X_{F^{\prime}}$ have the same definable properties (such as continuity and closedness) as $f_{F}$ and $X_{F}$. For convenience of notation, in the statement of Theorem 6.4, below, the term subanalytic function (respectively subanalytic set) refers to the family of functions $f_{F}$ (respectively sets $X_{F}$ ) defined uniformly by $\varphi$ (respectively $\psi$ ) in all models $F$ of $\mathcal{T}_{a n}(S(K))$. The Łojasiewicz data defined in Theorem 6.4 depends only on the corresponding $L_{a n}(S(K))$-formulas and not on the particular model.

(6.4) Theorem (Lojasiewicz Inequalities). $\quad$ (i) Let $f$ be a continuous subanalytic function with closed domain and zero set $Z$. Let $d(x, Z)$ be the distance from $x$ to $Z$ (put $d(x, \emptyset):=1$ ). Then there are $\alpha \in \mathbb{Q}_{+}=\mathbb{Q} \cap[0, \infty)$ and $c \in \sqrt{|K| \backslash\{0\}}$ such that

$$
|f(x)| \geq \operatorname{cd}(x, Z)^{\alpha}
$$

for all $x$ in the domain of $f$.

(ii) Let $f_{1}, \ldots, f_{n}$ be continuous subanalytic functions defined on the closed subanalytic set $X$. Then there are $\alpha \in \mathbb{Q}_{+}$and $c \in \sqrt{|K| \backslash\{0\}}$ such that for any $0<\varepsilon \leq 1$, if $x_{0} \in X$ satisfies

$$
\max _{1 \leq i \leq n}\left|f_{i}\left(x_{0}\right)\right| \leq c \varepsilon^{\alpha},
$$

there is an $x \in X$ with

$$
f_{1}(x)=\ldots=f_{n}(x)=0 \text { and }\left|x-x_{0}\right| \leq \varepsilon .
$$


(iii) Let $f$ and $g$ be continuous subanalytic functions defined on the closed subanalytic set $X$. Suppose that $g^{-1}(0) \subset f^{-1}(0)$. Then there are $\alpha \in \mathbb{Q}_{+}$and $c \in \sqrt{|K| \backslash\{0\}}$ such that

$$
|g(x)| \geq c|f(x)|^{\alpha}
$$

for all $x \in X$.

(iv) Let $X$ and $Y$ be closed subanalytic sets with $X \cap Y \neq \emptyset$. Then there are $\alpha \in \mathbb{Q}_{+}$and $c \in|K| \backslash\{0\}$ such that for any $0<\varepsilon \leq 1$, if $x_{0}$ satisfies

$$
d\left(x_{0}, X\right), d\left(x_{0}, Y\right) \leq c \varepsilon^{\alpha},
$$

then there is an $x \in X \cap Y$ with $\left|x-x_{0}\right| \leq \varepsilon$.

In each of (i)-(iv), let $\alpha_{0}$ be the infimum of the set of all $\alpha$ for which there is $a c \neq 0$ satisfying the respective statement. Then $\alpha_{0} \in \mathbb{Q}_{+}$and there corresponds $a c \neq 0$ satisfying the statement. Furthermore, fixing $\alpha=\alpha_{0}$, either the statement is satisfied for all $c \in \sqrt{|K| \backslash\{0\}}$ or the set of all $c \in|K| \backslash\{0\}$ satisfying the statement is bounded and has a maximal element $c_{0}$. The pair $\left(\alpha_{0}, c_{0}\right)$ is called the Łojasiewicz data.

Proof. (i) Define

$$
\mu(\delta):= \begin{cases}1 & \text { if } d(x, Z)<\delta \text { for all } x, \\ \inf \{|f(x)|: d(x, Z) \geq \delta\} & \text { otherwise. }\end{cases}
$$

By Theorem 5.2, there is a quantifier-free $L_{a n}^{D}$-formula $\varphi(\delta, \mu)$ such that $\mu=\mu(\delta)$ if, and only if, $\varphi(\delta, \mu)$ is true. Since $\delta$ and $\mu$ are both of the value semi-group sort, $\mu(\delta)$ must be piecewise defined on finitely many intervals by equations of the form

$$
\mu^{a}=\gamma \delta^{b}
$$

where $\gamma \in|K| \backslash\{0\}$ and $a, b \in \mathbb{N}, a \neq 0$. One such equation must hold for all $\delta$ less than some $\delta_{0} \in|K| \backslash\{0\}$. Put

$$
\alpha:=b / a
$$

(note that $\alpha_{0}$ must equal $\alpha$ ), and put

$$
c:=\inf _{\delta \in\left|F^{\circ}\right|} \mu(\delta) \cdot \delta^{-\alpha} .
$$

By Lemma 6.3,

$$
0 \neq \inf _{\delta \geq \delta_{0}} \mu(\delta) \cdot \delta^{-\alpha},
$$

and for all $\delta<\delta_{0}$,

$$
0 \neq \mu(\delta) \cdot \delta^{-\alpha}=\gamma^{1 / a} .
$$

We have $\mu(\delta) \geq c \cdot \delta^{\alpha}$ for all $\delta$. Since $c$ is quantifier-free $L(K)$-definable, $c \in$ $\sqrt{|K| \backslash\{0\}}$, as required.

(ii) This follows by applying (i) to

$$
f(x):=\max _{1 \leq i \leq n}\left|f_{i}(x)\right| .
$$

(iii) Consider the $L_{a n}$-definable function

$$
\mu(\delta):= \begin{cases}1 & \text { if }|f(x)|<\delta \text { for all } x, \\ \inf \{|g(x)|:|f(x)| \geq \delta\} & \text { otherwise, }\end{cases}
$$

and argue as in (i). 
(iv) Take

and apply (ii).

$$
f_{1}(x):=d(x, X), f_{2}(x):=d(x, Y)
$$

Let $\varphi\left(\xi_{1}, \ldots, \xi_{m}, \eta\right)$ be an $L_{a n}^{D}(U)$-formula, and suppose that $\varphi$ defines a (partial) function $f_{F}: F^{m} \rightarrow F$ in each model $F$ of $\mathcal{T}_{a n}(U)$. We refer to the family $\left\{f_{F}\right\}$ as a uniformly defined family of subanalytic functions. Such a family is called continuous if each member is continuous, and similarly for other definable properties. An $L_{a n}^{D}(U)$-formula $\psi\left(\xi_{1}, \ldots, \xi_{m}\right)$ defines a subset $X \subset F^{m}$ in each model $F$ of $\mathcal{T}_{a n}(U)$. The family $\left\{X_{F}\right\}$ is called a uniformly defined family of subanalytic sets. Such a family is called closed if each $X_{F}$ is closed, and similarly for other definable properties. Note that it is sufficient to verify these properties for each prime model of $\mathcal{T}_{a n}(U)$; see Corollaries 4.4 and 4.5 .

(6.5) Theorem (Uniform Łojasiewicz Data). Let $\chi=(p, q)$ be a pair consisting of a characteristic and residue characteristic. In each Eojasiewicz inequality (i)(iv), assume that the subanalytic functions and sets are uniformly defined, and let $\left(\alpha_{\chi}, c_{\chi}\right)$ be the corresponding Lojasiewicz data obtained in the models $F^{\circ}$ of $\mathcal{T}_{\text {an }}(U)$ with characteristic $p$ and residue characteristic $q$. There is a $q_{0}$ such that for every $\chi=(p, q)$ with $q \geq q_{0},\left(\alpha_{\chi}, c_{\chi}\right)=\left(\alpha_{0}, c_{0}\right)$, the Eojasiewicz data in equicharacteristic zero. Furthermore, each $\alpha_{\chi} \in \mathbb{Q}_{+}, c_{\chi} \in \mathbb{Q}$.

Proof. Since all the functions and sets are uniformly definable, we may replace use of Theorem 5.2 with the Uniform Elimination Theorem 4.2 in the proof of Theorem 6.4.

(6.6) Remark. There are parameterized versions of Theorems 6.4 and 6.5. Let $\varphi\left(\xi_{1}, \ldots, \xi_{m}, \eta_{1}, \ldots, \eta_{n+1}\right)$ be an $L_{a n}^{D}(S)$ formula such that $\varphi(\bar{\xi}, \eta)$ defines the graph of a function $f_{\bar{\xi}}$ for each $\bar{\xi} \in\left(F^{\circ}\right)^{m}$. We call the family $\left\{f_{\bar{\xi}}\right\}$ continuous if each $f_{\bar{\xi}}$ is continuous, and similarly for other definable properties of functions. We treat parameterized families of sets and their definable properties analogously. In each Łojasiewicz inequality of Theorem 6.4(i)-(iv), assume the functions and sets are parameterized families, and let $\left\{\left(\alpha_{\bar{\xi}}, c_{\bar{\xi}}\right)\right\}$ be the corresponding Łojasiewicz data. Applying the proof of Theorem 6.4 yields finitely many $L_{a n}^{D}(S)$-terms $t_{j}(\xi)$, finitely many nonnegative integers $s_{j}$ and an integer $r>0$ such that for each $\bar{\xi}$ there is some $j$ with $\left(\alpha_{\bar{\xi}}, c_{\bar{\xi}}\right)=\left(s_{j} / r,\left|t_{j}(\bar{\xi})\right|^{1 / r}\right)$. If, in addition, the parameterized families are given by $L_{a n}^{D}(U)$-formulas, we obtain the parameterized analogue of Theorem 6.5 .

\section{Closure of Semi-Analytic Sets}

Several different classes of rigid semianalytic sets have been studied in the literature. Some of them have been shown to be closed under the operation of topological closure. For example, using his valuation spectra, Huber $[\mathrm{H}]$ shows that the class of sets defined by equations and inequalities among strictly convergent power series has that property. Using resolution of singularities, Schoutens [S5] independently proved the same result in the characteristic zero case. In this section, we use the Łojasiewicz inequalities of the previous section to give an elementary and uniform proof that the closure of a semianalytic set is semianalytic. This proof yields the additional information that the closure may be described by functions belonging to the $\mathbb{Z}$-algebra generated by the functions in $\varphi$ and their (Hasse) derivatives. 
The setting of $\mathrm{G}$-topological spaces $(X, \mathcal{T}(X), \operatorname{Cov}(X))$ provides a common framework for the various classes of semianalytic sets. Examples of such spaces are: (i) an affinoid variety $X$ with the class of admissible open sets $\mathcal{T}(X)$ taken to be the class of rational domains, and the family of admissible covers $\operatorname{Cov}(X)$ taken to be the family of finite covers by rational domains, (ii) the "wobbly" quasi-affinoid variety $X=\left(F^{\circ}\right)^{m} \times\left(F^{\circ \circ}\right)^{n}$ with the class of admissible open sets $\mathcal{T}(X)$ taken to be the class of $R$-domains, and the family of admissible covers $\operatorname{Cov}_{W}(X)$ taken to be the family of finite covers by $R$-domains, and (iii) the "rigid" quasi-affinoid variety $\left(X, \mathcal{T}(X), \operatorname{Cov}_{R}(X)\right)$ with $X$ and $\mathcal{T}(X)$ as in (ii), but $\operatorname{Cov}_{R}(X)$ restricted to those covers that have suitable overlap; see LR6]. Suppose $X$ is a G-topological space with a pre-sheaf $\mathcal{O}_{X}$ of algebras of (analytic) functions. For example, if $X$ is an affinoid variety and $U$ is a rational subdomain of $X, \mathcal{O}_{X}(U)$ is the naturally associated affinoid algebra [BGR, Section 8.2.1], and if $X$ is a quasi-affinoid variety and $U$ is an $R$-subdomain, $\mathcal{O}_{X}(U)$ is the naturally associated quasi-affinoid algebra [LR4, Section 5.3].

(7.1) Definition. Let $F$ be a non-Archimedean valued field, let $X$ be a $\mathrm{G}$-topological space and let $\mathcal{O}_{X}$ be a pre-sheaf of rings of (analytic) functions into $F$. Let $U$ be an admissible open set of $X$. A globally semianalytic subset of $U$ is a set defined by a finite Boolean combination of conditions of the forms

$$
f(\xi)=0,|f(\xi)|<|g(\xi)| \text { or }|f(\xi)| \leq|g(\xi)|
$$

where $f, g \in \mathcal{O}_{X}(U)$. A semianalytic subset of $U$ is a set $Y \subset U$ such that there is an admissible cover $\{V\}$ of $U$ for which each set $Y \cap V$ is a globally semianalytic subset of $V$.

(7.2) Remark. One could define the class of semianalytic subsets of an affinoid $X$ to be the finite Boolean combinations of sets $Y_{i}$ that are globally semianalytic in some rational domain $U_{i} \subset X$ (see, e.g., [S5]). By modifying the topology of $X$ and the sheaf $\mathcal{O}_{X}$, this too fits the above definition: By $\mathcal{T}(X)$ denote the collection of rational subdomains of $X$. Let $\mathcal{T}^{\prime}(X)$ consist of all finite Boolean combinations of elements of $\mathcal{T}(X)$; these are the admissible open sets of a finer $G$-topology on $X$. Let $\operatorname{Cov}^{\prime}(X)$ consist of all finite covers of $X$ by members of $\mathcal{T}^{\prime}(X)$; these are the admissible covers. Finally, for $U \in \mathcal{T}^{\prime}(X)$, define

$$
\mathcal{O}_{X}^{\prime}(U):=\lim _{U \subset V \in \mathcal{T}(X)} \mathcal{O}_{X}(V)
$$

The next theorem shows that the closure of a semianalytic set is semianalytic.

(7.3) Theorem. Let $X$ be a subset of $\left(F^{\circ}\right)^{m} \times\left(F^{\circ \circ}\right)^{n}$ defined by a quantifier-free $L_{a n}(S)$-formula $\varphi$. Then $\bar{X}$, the closure of $X$, is also defined by a quantifier-free $L_{a n}(S)$-formula $\psi$. Moreover, $\psi$ can be chosen so that the functions occurring in $\psi$ belong to the $\mathbb{Z}$-algebra generated by the functions in $\varphi$ and their (Hasse) derivatives. Hence if a subring $\mathcal{H} \subset S$ is closed under derivatives and if $\varphi$ is a quantifier-free $L_{a n}(\mathcal{H})$-formula, then so is the corresponding $\psi$. This applies in particular when $\mathcal{H}=\bigcup_{m} T_{m}$ and when $\mathcal{H}=\mathcal{E}$, the existentially definable separated power series of [LR5], to yield in the respective categories that the closure of a semianalytic set is semianalytic. 
Proof. Since the closure of a finite union is the union of the closures, it is sufficient to consider formulas $\varphi$ of the form

$$
\bigwedge_{i=1}^{L} f_{i}(x)=0 \wedge \bigwedge_{i=1}^{M}\left|g_{i}(x)\right| \leq\left|h_{i}(x)\right| \wedge \bigwedge_{i=1}^{N}\left|g_{i}^{\prime}(x)\right|<\left|h_{i}^{\prime}(x)\right|,
$$

where the Gauss norm of all functions occurring in $\varphi$ is at most 1 . We shall show how to produce a quantifier-free formula $\psi$ that says " $y \in \bar{X}$."

Note that

$$
\varphi \leftrightarrow\left[\varphi \wedge\left(h_{i_{0}}(x)=0\right)\right] \vee\left[\varphi \wedge\left(0<\left|h_{i_{0}}(x)\right|\right)\right] .
$$

The first disjunct is equivalent to a formula of the same form as $\varphi$, where $M$ has decreased. Hence by induction on $M$, we may replace $\varphi$ by its conjunction with $\bigwedge\left(0<\left|h_{i}(x)\right|\right)$; in other words, we may assume that all the $h_{i}$ occur among the $h_{i}^{\prime}$.

Consider the function

$$
\mu(y, z):=\sup _{\substack{|x| \leq|z| \\ y+x \in X}} \min _{i}\left\{\left|h_{i}^{\prime}(y+x)\right|\right\} .
$$

It is a continuous, subanalytic function on the closed subanalytic set $\bar{X} \times F^{\circ}$. The zero set of $\mu$ is contained in $\bar{X} \times\{0\}$ because if $a \in X, \min _{i}\left|h_{i}^{\prime}(a)\right| \neq 0$, and if $z \neq 0$ and $y \in \bar{X}$, there is an $a \in X$ such that $|y-a|<|z|$. By Theorem 6.4(iii) applied to the pair of functions $\mu$ and $|z|$, there is an $\alpha \in \mathbb{N}$ such that for all $|z|$ sufficiently small,

$$
|\mu(y, z)| \geq|z|^{\alpha-1} .
$$

By Theorem 6.4(ii) applied to $f_{1}, \ldots, f_{L}$, there is a $\beta \in \mathbb{N}$ such that for any $\varepsilon>0$, if

$$
\max _{i}\left|f_{i}(u)\right|<\varepsilon^{\beta},
$$

there exists a $v$ with $|v|<\varepsilon$ such that

$$
f_{1}(u+v)=\ldots=f_{L}(u+v)=0 .
$$

We show that the following formula defines $\bar{X}$ :

$$
\begin{gathered}
\forall \varepsilon>0 \exists x\left[(0 \neq x) \wedge(|x|<\varepsilon) \wedge \bigwedge_{i=1}^{L}\left(\left|f_{i}(y+x)\right|<|x|^{\beta \alpha}\right)\right. \\
\wedge \bigwedge_{i=1}^{N}\left(\left|h_{i}^{\prime}(y+x)\right| \geq|x|^{\alpha}\right) \wedge \bigwedge_{i=1}^{M}\left(\left|g_{i}(y+x)\right| \leq\left|h_{i}(y+x)\right|\right) \\
\left.\wedge \bigwedge_{i=1}^{N}\left|g_{i}^{\prime}(y+x)\right|<\left|h_{i}^{\prime}(y+x)\right|\right] .
\end{gathered}
$$

Indeed, if $y \in \bar{X}$ and $\varepsilon>0$ there is some $a \in X$ with $|y-a|<\varepsilon$, which by (*) can be chosen to satisfy

$$
\min _{i}\left\{\left|h_{i}^{\prime}(a)\right|\right\} \geq \varepsilon^{\alpha}>|y-a|^{\alpha} .
$$

Thus, setting $x:=a-y$, we see that $(* *)$ is satisfied. Conversely, suppose $y$ satisfies the formula, let $\varepsilon>0$, and let $x$ be guaranteed by $(* *)$. Then by the definition of $\beta$, there is a $v$ with $|v|<|x|^{\alpha}$ and

$$
f_{1}(y+x+v)=\ldots=f_{i}(y+x+v)=0 .
$$


Since the $h_{i}$ are all among the $h_{i}^{\prime}$, since all the functions have Gauss norm at most 1 and hence satisfy a Lipschitz condition with constant at most 1, it follows from the inequalities in $(* *)$ that $y+x+v \in X$, as required.

It remains to eliminate the quantifiers in $(* *)$. By the algebraic elimination theorem, it suffices to show that the conditions in $(* *)$ are equivalent to conditions that are polynomial in $x$. Note that

$$
\begin{aligned}
\left|f_{i}(y+x)\right|<|x|^{\beta \alpha} & \Leftrightarrow\left|\sum_{\nu} D_{\nu} f_{i}(y) x^{\nu}\right|<|x|^{\beta \alpha} \\
& \Leftrightarrow\left|\sum_{|\nu| \leq \beta \alpha} D_{\nu} f_{i}(y) x^{\nu}\right|<|x|^{\beta \alpha},
\end{aligned}
$$

which is a polynomial condition in $x$. The other clauses may be handled similarly, expanding each function as a power series in $x$. Since $|x|^{\alpha} \leq\left|h_{i}^{\prime}(y+x)\right|$, these series may be truncated at order $\alpha$ in $x$ to yield an equivalent system of inequalities that are polynomial in $x$. The algebraic elimination theorem produces an equivalent quantifier-free formula with the property that all of its terms belong to the $\mathbb{Z}^{-}$ algebra generated by the functions $f_{i}, g_{i}, g_{i}^{\prime}, h_{i}, h_{i}^{\prime}$ and their Hasse derivatives.

Note that $U$ is closed under Hasse derivatives, that the Eojasiewicz inequalities of Theorem 6.4 have a uniform version, Theorem 6.5, and that the algebraic elimination theorem is uniform; thus each step of the proof of Theorem 7.3 holds in the uniform setting. The following uniform version of Theorem 7.3 is an immediate consequence.

(7.4) Corollary. Let $\varphi$ be a quantifier-free $L_{a n}(U)$-formula. For any model $F^{\circ}$ of $\mathcal{T}_{\text {an }}(U)$, let $X_{F}$ be the globally semianalytic set defined by $\varphi$ and let $\bar{X}_{F}$ be its closure. Then there is a quantifier-free $L_{a n}(U)$-formula $\psi$ such that $\psi$ defines $\bar{X}_{F}$ in each $F^{\circ}$.

Making use of the parameterized Łojasiewicz inequalities of Remark 6.6, we obtain the following.

(7.5) Corollary. Let $\varphi(\xi, \eta)$ be a quantifier-free $L_{a n}^{D}$-formula in which $D$ is not applied to any term involving $\eta$. Then there is a quantifier-free $L_{a n}^{D}$-formula $\psi(\xi, \eta)$ in which $D$ is not applied to any term involving $\eta$ such that for each $\bar{\xi} \in\left(F^{\circ}\right)^{m}, \psi(\bar{\xi}, \eta)$ defines the closure of the set defined by $\varphi(\bar{\xi}, \eta)$.

\section{Curve selection}

In this section, we prove curve selection for rigid subanalytic sets, a result which has already been established by Huber $[\mathrm{H}]$. The proof of Theorem 8.1 proceeds by a reduction to curve selection for semianalytic sets. Lemma 8.2 reduces the problem to treating the open and one-dimensional semianalytic cases. Lemma 8.3 treats the one-dimensional case, and Lemma 8.4 reduces the open case to the one-dimensional semi-algebraic case (which can be handled by Lemma 8.3).

Let $F$ be an algebraically closed complete valued field extension of $K$.

(8.1) Theorem. Let $X \subset\left(F^{\circ}\right)^{m}$ be subanalytic and let $a \in \bar{X}$, the closure with respect to the metric topology on $F$. Then there is an analytic curve $\xi=\xi(t) \in$ $(F\{t\})^{m}$, where $F\{t\}$ is the ring of convergent power series over $F$ in the variable $t$, such that $\xi(0)=a$ and $\xi(t) \in X$ for $t \neq 0$ and $|t|$ sufficiently small. 
Proof. By the Quantifier Simplification Theorem 5.2, there is a semianalytic subset $Y \subset\left(F^{\circ}\right)^{m+n}$ such that $X=\pi(Y)$, where $\pi:\left(F^{\circ}\right)^{m+n} \rightarrow\left(F^{\circ}\right)^{m}$ is the projection onto the first $m$ coordinates. For $b$ in the closed subanalytic set $\{a\} \times$ $\left(F^{\circ}\right)^{n}$, consider the continuous subanalytic function $\mu(b):=\inf _{y \in Y}|b-y|$. Since $\inf _{b \in\{a\} \times\left(F^{\circ}\right)^{n}} \mu(b)=0$, by Lemma 6.3 , there is some $b_{0} \in \bar{Y}$ such that $\pi\left(b_{0}\right)=a$. The theorem now follows from Lemma 8.2.

(8.2) Lemma. Let $F$ be an algebraically closed, complete valued field extension of $K$. Let $X \subset\left(F^{\circ}\right)^{m}$ be semianalytic and let $a \in \bar{X}$. Then there is an analytic curve $\xi=\xi(t) \in(F\{t\})^{m}$ such that $\xi(0)=a$ and $\xi(t) \in X$ for $t \neq 0$ and $|t|$ sufficiently small.

Proof. We may assume $a=0$. First, suppose $0 \notin Y$, the closure of the interior of $X$. Then there is a rational polydisc $B \ni 0$ such that $B$ does not meet the interior of $X$. It suffices to prove the lemma for $B \cap X$ in place of $X$. Note that $\operatorname{dim} B \cap X<m$. By shrinking $B$, we may assume that $B \cap X$ is contained in the zero set of some $f \in F\{\xi\} \backslash\{0\}$. After a change of variables, we may assume that $f$ is regular in $\xi_{m}$ of some degree. By the local Weierstrass Preparation Theorem and Algebraic Elimination, it follows that $\pi(B \cap X) \subset\left(F^{\circ}\right)^{m-1}$ is semianalytic. By induction on $m$, there is an ideal $I^{\prime}$ of $F\{\xi\}$ such that (shrinking $B$ if necessary) 0 belongs to the closure of $V\left(I^{\prime}\right) \cap \pi(B \cap X)$, and $\operatorname{dim} V\left(I^{\prime}\right)=1$.

Let $I$ be the ideal of $F\{\xi\}$ generated by $f$ and the elements of $I^{\prime}$. Then $\operatorname{dim} V(I)=1$ and 0 belongs to the closure of $V(I) \cap B \cap X$. This is a semianalytic subset of $X$ of dimension 1. An application of Lemma 8.3 completes the proof in this case.

It remains to treat the case that $0 \in Y$, which, by Theorem 7.3 is semianalytic. This is taken care of by Lemma 8.4.

(8.3) Lemma. Let $X \subset\left(F^{\circ}\right)^{m}$ be semianalytic. Suppose $\operatorname{dim} X \leq 1$, and let $a \in \bar{X}$. Then there is an analytic curve $\xi=\xi(t) \in(F\{t\})^{m}$ such that $\xi(0)=a$ and $\xi(t) \in X$ for $t \neq 0$ and $|t|$ sufficiently small.

Proof. We may assume that $a=0$. Let $I$ be the ideal of all $f \in F\{\xi\}$ that vanish on $X$; then $F\{\xi\} / I$ has Krull dimension 1. Since $F\{\xi\}$ is Noetherian and since the closure of a finite union is the union of the closures, we may replace $I$ by one of its prime divisors; i.e., we assume that $I$ is prime. By Noether Normalization, after a change of variables the inclusion $\sigma: F\left\{\xi_{1}\right\} \rightarrow F\{\xi\} / I$ is finite.

Let $f=\sum a_{i} \xi_{1}^{i} \in F\left\{\xi_{1}\right\} \backslash\{0\}$ and define $|f|:=\left(2^{-d},\left|a_{d}\right|\right)$, where $a_{d} \neq 0$ and $a_{i}=0$ for $i<d$. Order $|F\{\xi\}|$ lexicographically. Note that $|\cdot|$ extends the valuation on $F$. It follows from the Weierstrass Preparation Theorem, Corollary 3.3, as in the proof of Theorem 5.9(ii) that $\mathcal{F}^{\circ}:=\left(F\left\{\xi_{1}\right\},|\cdot|\right)$ is a Henselian valuation ring.

Since $X$ is semianalytic, we may assume that it is defined by a quantifier-free $L_{a n}(F)$-formula $\varphi$. Furthermore, by Weierstrass Division, we may write all functions occurring in $\varphi$ as polynomials in $\xi_{2}, \ldots, \xi_{m}$ with coefficients in $F\left\{\xi_{1}\right\}$. Since $\mathcal{F}_{\text {alg }}^{\circ}$ is a model of $\mathcal{T}_{\text {an }}(F)$, since $\xi_{1}$ belongs to the projection of $X$ (because the projection is a Boolean combination of $F$-rational discs and $\left.\left|\xi_{1}\right|<|F \backslash\{0\}|\right)$, for at least one of the conjugates of $\left(\xi_{2}, \ldots, \xi_{m}\right), \varphi(\xi)$ must hold. Since $\mathcal{F}^{\circ}$ is Henselian, the valuation on $\mathcal{F}^{\circ}$ extends uniquely $\mathrm{E}$ to $\mathcal{F}_{\text {alg }}$, and hence for all conjugates of $\left(\xi_{2}, \ldots, \xi_{m}\right), \varphi(\xi)$ holds. It follows that there is an $F$-rational disc $B \ni 0$ such that $X \cap B=V(I) \cap B$. Therefore, it suffices to find an analytic curve $\xi=\xi(t) \in(F\{t\})^{m}$ such that $\xi(0)=0$ and $\xi(t) \in V(I)$ for $|t|$ sufficiently small. 
Let $N$ be the integral closure of $F\{\xi\} / I$ in its field of fractions. By BGR. Proposition 6.1.2.4], $N$ is finite over $F\{\xi\} / I$. Let $\mathfrak{m}$ be a maximal ideal of $N$; the localization $N_{\mathfrak{m}}$ is a one-dimensional Noetherian, normal, local ring. By Mat, Theorem 11.2], it is therefore a discrete valuation ring. Let $t$ be a generator of its maximal ideal; we have a natural map $N_{\mathfrak{m}} \rightarrow F\{t\}$. The composition $F\{\xi\} / I \rightarrow$ $N_{\mathfrak{m}} \rightarrow F\{t\}$ yields the desired curve $\xi$.

(8.4) Lemma. Let $X \subset\left(F^{\circ}\right)^{m}$ be an open semianalytic set and let $a \in \bar{X}$. Then there is a rational polydisc $B$ and a one-dimensional algebraic variety $Z$ such that $a$ is in the closure of $B \cap Z \cap X$. In particular, Lemma 8.3 yields analytic curve selection for $X$ at $a$.

Proof. We may assume that $a=0$. Let $\varphi$ be a quantifier-free $L_{a n}(F)$-formula defining $X$. Since $X$ is open, by breaking into finitely many open pieces, we may assume that $\varphi$ is

$$
\left|f_{1}(\xi)\right| \bowtie_{1} \cdots \bowtie_{n-1}\left|f_{n}(\xi)\right|
$$

where $\bowtie_{i} \in\{<, \leq\}$. We may assume $f_{1}(0)=\cdots=f_{n}(0)=0$.

Define $\mu(\xi):=\sup \left\{\left|f_{i}(\eta)\right|: \eta \in X,|\eta| \leq|\xi|\right\}$. Note that $\mu^{-1}(0)=\{0\} \subset f_{1}^{-1}(0)$. So by Theorem $6.4\left(\right.$ iii), there are $\alpha \in \mathbb{N}, c \in F$ such that $\mu(\xi) \geq|c \xi|^{\alpha}$. The set defined by

$$
\varphi \wedge|c \xi|^{\alpha+1}<\left|f_{1}(\xi)\right|
$$

is open, non-empty and has 0 in its closure.

Write each $f_{i}=\sum a_{i \nu} \xi^{\nu}$; in $(*)$, we may replace each $f_{i}$ by $\sum_{|\nu| \leq \alpha} a_{i \nu} \xi^{\nu}$. In particular, we may assume that $\varphi$ is a quantifier-free $L(F)$-formula (i.e., all variables occur polynomially).

By algebraic elimination, there is some rational disc $0 \in B \subset F^{\circ}$ of radius $\epsilon$ such that $B \subset \pi(X)$, where $\pi:\left(F^{\circ}\right)^{m} \rightarrow F^{\circ}$ is coordinate projection. Let $a_{i j}, z$ be new constant symbols, and let $p_{i}(x)$ be an enumeration of all polynomials in one variable, $x$, with coefficients in $F[z]$ and suppose the degree of $p_{i}$ is $n_{i}$. Let $\gamma_{i}$ be the formula that asserts that all the zeroes of $p_{i}$ are among $a_{i 1}, \ldots, a_{i n_{i}}$, and let $T_{F}$ be the theory of $F$ (i.e., all $L$-sentences true in $F$ ). Consider the theory

$$
T:=T_{p} \cup\left\{\gamma_{i}\right\} \cup\left\{\neg \varphi\left(z, a_{i_{1} j_{1}}, \ldots, a_{i_{n-1} j_{n-1}}\right\} \cup\{|z|<\epsilon\} .\right.
$$

Suppose $\left(F^{\prime}\right)^{\circ}$ were a model of $T$. Taking the submodel $\mathcal{F}:=F(z)_{a l g}$, we see that

$$
\forall x_{1}, \ldots, x_{n-1} \neg \varphi\left(z, x_{1}, \ldots, x_{n-1}\right)
$$

holds in $\mathcal{F}$, which, by model completeness, contradicts the definition of $\epsilon$. By modeltheoretic compactness, there is a finite set of polynomials $p_{1}, \ldots, p_{k}$, such that in every algebraically closed, valued field $F^{\prime} \supset F$ and for every $z \in F^{\prime}$ with $|z|<\epsilon$ there are $a_{1}, \ldots, a_{n-1}$ among the zeros of $p_{1}, \ldots, p_{k}$ such that $\varphi\left(z, a_{1}, \ldots, a_{n-1}\right)$ holds. Let $P(x):=\prod_{i=1}^{k} p_{i}(x)$. Then putting $Z:=V\left(P\left(\xi_{1}\right), \ldots, P\left(\xi_{n}\right)\right)$ satisfies the lemma.

(8.5) Corollary. Let $F^{\circ} \vDash \mathcal{T}_{a n}(U)$ (respectively $T_{a n}(K)$ ) and let $X_{F}$ be a subanalytic subset of $\left(F^{\circ}\right)^{m}$ defined by an $L_{a n}(U)$-formula (respectively $L_{a n}(K)$-formula) $\varphi$ with parameters from $F^{\circ}$. Let $a \in \bar{X}_{F}$. There is an $L_{a n}(U)$-formula (respectively $L_{a n}(K)$-formula) $\psi$ with parameters from $F$ which defines a one-dimensional variety near $a$ and an $F$-rational ball $B \ni$ a such that $a$ is in the closure of $B \cap X_{F} \cap Y_{F}$. 
(8.6) Remark. Similar arguments can be used to prove a parametrized analogue of Theorem 8.1.

\section{REFERENCES}

[Ba1] W. Bartenwerfer, Der allgemeine Kontinuitätssatz für $k$-meromorphe Funktionen im Dizylinder, Math. Ann. 191 (1971), 196-234. MR0287035 (44:4242)

[Ba2] W. Bartenwerfer, Die Beschränktheit der Stückzahl der Fasern K-analytischer Abbildungen, J. Reine Angew. Math. 416 (1991), 49-70. MR1099945 (92e:32017)

[BGR] S. Bosch, U. Güntzer and R. Remmert, Non-Archimedean Analysis, Springer-Verlag, 1984. MR0746961 (86b:32031)

[D1] J. Denef, p-adic semi-algebraic sets and cell decomposition, J. Reine Angew. Math. 369 (1987), 991-1008. MR0850632 (88d:11030)

[D2] - Arithmetic and geometric applications of quantifier elimination for valued fields, Model Theory, Algebra and Geometry, vol. 39, MSRI Publications, 2000, pp. 173-198. MR 1773707 (2001e:03063)

[DD] J. Denef and L. van den Dries, $p$-adic and real subanalytic sets, Ann. Math. 128 (1988), 79-138. MR0951508 (89k:03034)

[E] O. Endler, Valuation Theory, Springer-Verlag, 1972. MR0357379 (50:9847)

$[\mathrm{H}] \quad$ R. Huber, On semianalytic subsets of rigid analytic varieties, Geometriae Dedicata $\mathbf{5 8}$ (1995), 291-311. MR1358458 (96k:32069)

[Kap1] I. Kaplansky, Maximal fields with valuations, Duke Math. J. 9 (1942), 312-321. MR0006161 (3:264d)

[Kap2] , Maximal fields with valuations II, Duke Math. J. 12 (1945), 243-248. MR0012276 $(7: 3 \mathrm{~d})$

[L] L. Lipshitz, Rigid subanalytic sets, Amer. J. Math. 115 (1993), 77-108. MR1209235 (94d:32047)

[Liu] N. Liu, Analytic cell decomposition and the closure of p-adic semianalytic sets, JSL 62 (1997), 285-303. MR1450524 (98h:03039)

[LR1] L. Lipshitz and Z. Robinson, Rigid subanalytic subsets of the line and the plane, Amer. J. Math. 118 (1996), 493-527. MR1393258 (97e:14067)

[LR2] _ Rigid subanalytic subsets of curves and surfaces, J. London Math. Soc. (2) 59 (1999), 895-921. MR.1709087 (2000j:32037)

[LR3] - One-dimensional fibers of rigid subanalytic sets, J. Symbolic Logic 63 (1998), 83-88. MR:1610778 (98m:32049)

[LR4] Rings of separated power series, Astérisque 264 (2000), 3-108. MR1758887 (2001g:32017)

[LR5] Model completeness and subanalytic sets, Astérisque 264 (2000), 109-126.

[LR6] - Quasi-affinoid varieties, Astérisque 264 (2000), 127-149.

[LR7] Dimension theory and smooth stratification of rigid subanalytic sets, Logic Conference '98 (S. Buss, P. Hájek and P. Pudlak, eds.); Lecture Notes in Logic, vol. 13, A.S.L., 2000, pp. 302-315. MR1743267 (2002b:32014)

[Mac1] A. Macintyre, On definable subsets of p-adic fields, J. Symbolic Logic 41 (1976), 605-610. MR0485335 (58:5182)

[Mac2] , Rationality of $p$-adic Poincaré series: uniformity in p, Ann. Pure Appl. Logic 49 (1990), 31-74. MR.1076249 (92b:11085)

[Mat] H. Matsumura, Commutative Ring Theory, Cambridge University Press, 1989. MR1011461 (90i:13001)

[Pas] J. Pas, Uniform p-adic cell decomposition and local zeta functions, J. Reine Angew. Math. 399 (1989), 137-172. MR1004136 (91g:11142)

[Pass] D.S. Passman, The Algebraic Structure of Group Rings, J. Wiley, 1977. MR0470211 (81d:16001)

[Po] B. Poonen, Maximally Complete Fields, L'Enseignement Math. 39 (1993), 87-106. MR1225257 (94h:12005)

[Ro] A. Robinson, Complete Theories, North-Holland, 1956. MR0075897 (17:817b)

[Rob1] Z. Robinson, Smooth points of p-adic subanalytic sets, Manuscripta Math. 80 (1993), 45-71. MR 1226596 (94h:32055) 
[Rob2] _ Flatness and smooth points of p-adic subanalytic sets, Ann. Pure Appl. Logic 88 (1997), 217-225. MR1600911 (99b:32050)

[S1] H. Schoutens, Rigid subanalytic sets, Comp. Math. 94 (1994), 269-295. MR 1310860 (96b:32043)

[S2] Rigid subanalytic sets in the plane, J. Algebra 170 (1994), 266-276. MR1302840 (96b:32041)

[S3] Uniformization of rigid subanalytic sets, Comp. Math. 94 (1994), 227-245. MR 1310858 (96b:32042)

[S4] , Blowing up in rigid analytic geometry, Bull. Belg. Math. Soc. 2 (1995), 399-417. MR 1355829 (96g:32053)

[S5] Closure of rigid semianalytic sets, J. Algebra 198 (1997), 120-134. MR1482978 (98m:14054)

[vdD] L. van den Dries, Analytic Ax-Kochen-Ersov Theorems, Contemporary Mathematics, vol. 131 part 3, AMS, 1992, pp. 379-398. MR1175894 (93i:03041)

[vHM] L. van den Dries, D. Haskell and D. Macpherson, One-dimensional p-adic subanalytic sets, J. London Math. Soc. (2) 59 (1999), 1-20. MR.1688485 (2000k:03077)

[W] V. Weispfenning, Quantifier Elimination and Decision Procedures for Valued Fields, Models and Sets, Aachen 1103 (1983), 1984, pp. 419-472. MR0775704 (86m:03059)

Department of Mathematics, Purdue University, West Lafayette, Indiana 47907

E-mail address: lipshitz@math.purdue.edu

Department of Mathematics, East Carolina University, Greenville, North Carolina 27858

E-mail address: robinsonz@mail.ecu.edu 